\title{
Vacua and walls of mass-deformed Kähler nonlinear sigma models on $\operatorname{Sp}(N) / U(N)$
}

\author{
Masato Arai, ${ }^{1, *}$ Anastasia Golubtsova, ${ }^{2,3, \dagger}$ Chanyong Park, ${ }^{4, \$}$ and Sunyoung Shin ${ }^{5, \S}$ \\ ${ }^{1}$ Faculty of Science, Yamagata University, Yamagata 990-8560, Japan \\ ${ }^{2}$ Bogoliubov Laboratory of Theoretical Physics, JINR, 141980 Dubna, \\ Moscow region, Russia \\ ${ }^{3}$ Dubna State University, Universitetskaya str. 19, Dubna 141980, Russia \\ ${ }^{4}$ Department of Physics and Photon Science, Gwangju Institute of Science and Technology, \\ Cheomdangwagi-ro 123, Buk-gu, Gwangju 61005, Republic of Korea \\ ${ }^{5}$ Institute of Basic Science, Sungkyunkwan University, Suwon 16419, Republic of Korea
}

(Received 5 April 2018; published 17 May 2018)

\begin{abstract}
We study vacua and walls of mass-deformed Kähler nonlinear sigma models on $S p(N) / U(N)$. We identify elementary walls with the simple roots of $\operatorname{USp}(2 N)$ and discuss compressed walls, penetrable walls, and multiwalls by using the moduli matrix formalism.
\end{abstract}

DOI: 10.1103/PhysRevD.97.105012

\section{INTRODUCTION}

Kähler and hyper-Kähler nonlinear sigma models are studied in Refs. [1-3]. Massive hyper-Kähler nonlinear sigma models have a potential that is proportional to the square of a triholomorphic Killing vector field of the hyper-Kähler target space [4]. Fixed points of the Killing vector field are realized as discrete vacua. It was shown that there exist $1 / 2$ supersymmetric kink solutions that interpolate the discrete vacua [5]. A more general potential is possible for a hyper-Kähler target space of quaternionic dimension 2 or more, and exact nonsingular solutions representing intersecting domain walls are constructed in Ref. [6]. Multidomain walls are studied in Ref. [7].

The moduli matrix formalism [8,9] was proposed to construct walls systematically in non-Abelian gauge theories with $\mathcal{N}=2$ supersymmetry in four-dimensional spacetime. The model considered in Refs. [8,9] becomes massive hyper-Kähler nonlinear sigma models on the cotangent bundle over the Grassmann manifold

\footnotetext{
*arai@sci.kj.yamagata-u.ac.jp

golubtsova@theor.jinr.ru

*yong21@gist.ac.kr

§sihnsy@skku.edu
}

Published by the American Physical Society under the terms of the Creative Commons Attribution 4.0 International license. Further distribution of this work must maintain attribution to the author(s) and the published article's title, journal citation, and DOI. Funded by SCOAP ${ }^{3}$.
$T^{*} G_{N_{F}, N_{C}}{ }^{1}$ when the gauge coupling is taken to be infinity. In this limit, multiwalls are constructed as well as single walls. Multiwalls are along one spatial direction, and their positions depend on moduli parameters and mass parameters. Walls can be compressed to single walls by changing moduli parameters in Abelian gauge theories and in nonAbelian gauge theories. These walls are called compressed walls. A distinguishing feature in the non-Abelian gauge theories is that walls can pass through each other [9]. Such walls are called penetrable walls. It was also shown in Ref. [9] that there is a bundle structure for nondegenerate masses so that the vacua and the walls are on the Kähler manifold.

The walls of Kähler nonlinear sigma models on $S O(2 N) / U(N)$ are studied in Refs. [10,11]. The Hermitian symmetric space $S O(2 N) / U(N)$ is realized as a quadric in the Grassmann manifold $G_{2 N, N}$ in accordance with Refs. [12,13]. As $S O(4) / U(2) \simeq \mathbf{C} P^{1}$ and $S O(6) / U(3) \simeq \mathbf{C} P^{3}$ [14], the nonlinear sigma models on $S O(2 N) / U(N)$ with $N=2$ and $N=3$ are actually Abelian gauge theories. The walls of the nonlinear sigma models on $S O(2 N) / U(N)$ with $N=2,3$ are studied in Ref. [10]. The walls of the nonlinear sigma models on $S O(2 N) / U(N)$ for any $N$ are studied in Ref. [11]. Penetrable walls, which are related to non-Abelian nature, appear in $N \geq 4$ cases. The vacua and the walls of $N \leq 7$ cases are presented in pictorial representations in which the vacua and elementary walls correspond to the vertices and the segments of the representations. It is shown that there is a recurrence of

\footnotetext{
${ }^{1} G_{N_{F}, N_{C}}=\frac{S U\left(N_{F}\right)}{S U\left(N_{C}\right) \times S U\left(N_{F}-N_{C}\right) \times U(1)}$.
} 
a two-dimensional diagram for each $N \bmod 4$ in the vacuum structures that are connected to the maximum number of elementary walls. The vacuum structures are proved by induction.

The purpose of this paper is to construct walls of mass-deformed Kähler nonlinear sigma models on $S p(N)$ / $U(N)^{2} \quad S p(N) \equiv U S p(2 N)$ or equivalently $\operatorname{Sp}(N)=$ $S p(N, \mathbf{C}) \cap U(2 N)$. Unlike $S U(N)$ or $S O(2 N)$, the lengths of the simple roots of $U S p(2 N)$ are different. Therefore, the operators for the compressed walls of the nonlinear sigma models on $\operatorname{Sp}(N) / U(N)$ should be newly defined. We discuss the definitions of the operators and show that some of the multiwalls can be compressed.

Since $S p(1) / U(1) \simeq \mathbf{C} P^{1} \simeq Q^{1}$ and $S p(2) / U(2) \simeq Q^{3}$ $[14],{ }^{3}$ the nonlinear sigma models on $S p(N) / U(N)$ with $N=1,2$ are Abelian theories. However, the nonlinear sigma models on $\operatorname{Sp}(N) / U(N)$ with $N \geq 3$ are nonAbelian theories, so there exist penetrable walls. We use the pictorial representations proposed in Ref. [11] to investigate the vacuum structures and the recurrence of two-dimensional diagrams to prove the vacuum structures that are connected to the maximum number of elementary walls by induction.
We follow the convention of Refs. [15,16] for the description of the root systems and corresponding Lie algebras. We also identify the elementary walls with the simple roots of $U S p(2 N)$ as it is done in Ref. [17]. In Sec. II, we discuss the nonlinear sigma models on $S p(N) / U(N)$ and the moduli matrix formalism. In Sec. III, we study walls of the Kähler nonlinear sigma models on $S p(N) / U(N)$ with $N \leq 6$. In Sec. IV, we study the vacuum structures that are connected to the maximum number of elementary walls. In Sec. $\mathrm{V}$, we make some observations about walls of the nonlinear sigma model on $S p(5) / U(5)$. In Sec. VI, we summarize our results. In the Appendix, we prove the vacuum structures that are connected to the maximum number of elementary walls.

\section{MODEL}

The Kähler nonlinear sigma models on $S O(2 N) / U(N)$ and $S p(N) / U(N)$ can be represented as quadrics in the Grassmann manifold $G_{2 N, N}$. The Lagrangian in four dimensions is written in the $\mathcal{N}=1$ superfield formalism $[13,14,18]$,

$$
\mathcal{L}=\int d^{4} \theta\left(\Phi_{a}{ }^{i} \bar{\Phi}_{i}{ }^{b}\left(e^{V}\right)_{b}{ }^{a}-\zeta V_{a}{ }^{a}\right)+\int d^{2} \theta\left(\Phi_{0}^{a b}\left(\Phi_{b}{ }^{i} J_{i j} \Phi^{T j}{ }_{a}\right)+(\text { H.c. })\right),
$$

where $\Phi$ is an $N \times 2 N$ chiral superfield with the flavor indices $i, j=1, \ldots, 2 N$ and the color indices $a, b=1, \cdots N, V$ is an $N \times N$ matrix vector superfield in the adjoint representation of $U(N)$, and $\Phi_{0}^{a b}$ is a chiral superfield under a symmetric representation of $U(N) . \zeta$ is the Fayet-Iliopoulos parameter, and we set $\zeta=1 . J_{i j}$ are invariant tensors defined by

$$
J= \begin{cases}\sigma^{1} \otimes I_{N}, & S O(2 N) / U(N) \\ i \sigma^{2} \otimes I_{N}, & S p(N) / U(N) .\end{cases}
$$

The superfields are written in terms of component fields:

$$
\begin{aligned}
\Phi_{a}{ }^{i}(y, \theta) & =\phi_{a}{ }^{i}(y)+\sqrt{2} \theta \psi_{a}{ }^{i}(y)+\theta \theta F_{a}{ }^{i}(y), \\
V_{a}{ }^{b}(x, \theta, \bar{\theta}) & =2\left(\theta \sigma^{m} \bar{\theta}\right) A_{m a}{ }^{b}(x)+i(\theta \theta)(\bar{\theta} \bar{\lambda})_{a}{ }^{b}(x)-i(\bar{\theta} \bar{\theta})(\theta \lambda)_{a}{ }^{b}(x)+(\theta \theta)(\bar{\theta} \bar{\theta}) D_{a}{ }^{b}(x), \\
\Phi_{0}^{a b}(y, \theta) & =\phi_{0}^{a b}(y)+\sqrt{2} \theta \psi_{0}^{a b}(y)+\theta \theta F_{0}^{a b}(y) .
\end{aligned}
$$

The mass-deformed Lagrangian is obtained by dimensional reduction [19]. The bosonic part of the Lagrangian in three dimensions is

$$
\begin{aligned}
\mathcal{L}= & -\left|\left(D_{\mu} \phi\right)_{a}{ }^{i}\right|^{2}-\left|i \phi_{a}{ }^{j} M_{j}{ }^{i}-i \Sigma_{a}{ }^{b} \phi_{b}{ }^{i}\right|^{2}+\left|F_{a}{ }^{i}\right|^{2}+\left(D_{a}{ }^{b} \phi_{b}{ }^{i} \bar{\phi}_{i}{ }^{a}-D_{a}{ }^{a}\right) \\
& \left.+\left(\left(F_{0}\right)^{a b} \phi_{b}{ }^{i} J_{i j} \phi^{T j}{ }_{a}+\phi_{0}^{a b} F_{b}{ }^{i} J_{i j} \phi_{a}^{T j}+\left(\phi_{0}\right)^{a b} \phi_{b}{ }^{i} J_{i j} F^{T j}{ }_{a}+\text { (H.c. }\right)\right),
\end{aligned}
$$

where the greek letter $\mu$ is a three-dimensional spacetime index. The covariant derivative is defined by $\left(D_{\mu} \phi\right)_{a}{ }^{i}=\partial_{\mu} \phi_{a}{ }^{i}-i A_{\mu a}{ }^{b} \phi_{b}{ }^{i}$. The last term (H.c.) stands for the Hermitian conjugate.

\footnotetext{
${ }^{2}$ The result of this paper is different than the result of Ref. [10]. In Ref. [10], we did not use the root system of $U S p(2 N)$ to analyze the vacua and the walls of the nonlinear sigma models on $S p(N) / U(N)$. In this paper, we identify the elementary wall operators with the simple root generators of $\operatorname{USp}(2 N)$ and find that the elementary wall operators in Ref. [10] are not correct. The result of this paper seems to be consistent with the result of Ref. [15] in which the kink monopoles are studied in similar models with $U S p(2 N)$ global symmetry.

${ }^{3} \mathbf{C} P^{N-1}=\frac{S U(N)}{S U(N-1) \times U(1)}$ and $Q^{N-2}=\frac{S O(2 N)}{S O(N-2) \times U(1)}$.
} 
The Cartan generators of $S O(2 N)$ and $U S p(2 N)$ are

$$
H_{I}=e_{I, I}-e_{N+I, N+I}, \quad(I=1, \cdots N),
$$

where $e_{I, I}\left(e_{N+I, N+I}\right)$ is a $2 N \times 2 N$ matrix of which the $(I, I)((N+I, N+I))$ component is $1[15,16]$. The mass matrix can be formulated as

$$
M=\vec{m} \cdot \vec{H},
$$

with vectors

$$
\begin{aligned}
\vec{m} & :=\left(m_{1}, m_{2} \cdots, m_{N}\right), \\
\vec{H} & :=\left(H_{1}, H_{2}, \ldots, H_{N}\right) .
\end{aligned}
$$

The mass matrix in the basis (2.5) is

$$
M=\sigma_{3} \otimes \operatorname{diag}\left(m_{1}, m_{2}, \ldots, m_{N}\right) .
$$

Since we are interested in generic mass parameters, we can set $m_{1}>m_{2}>\cdots>m_{N}$ without loss of generality.

Equations of motion for $D$ and $F$ yield the constraints for the Lagrangian (2.4)

$$
\phi_{a}{ }^{i} \bar{\phi}_{i}^{b}-\delta_{a}^{b}=0,
$$

$\phi_{a}{ }^{i} J_{i j} \phi^{T j}{ }_{b}=0, \quad($ Hermitian conjugate $)=0$.

We eliminate the auxiliary fields. The potential term of the model is

$$
V=\left|i \phi_{a}{ }^{j} M_{j}{ }^{i}-i \Sigma_{a}{ }^{b} \phi_{b}{ }^{i}\right|^{2}+4\left|\left(\phi_{0}\right)^{a b} \phi_{b}{ }^{i}\right|^{2} .
$$

The vacuum conditions are

$$
\begin{gathered}
\phi_{a}{ }^{j} M_{j}{ }^{i}-\Sigma_{a}{ }^{b} \phi_{b}{ }^{i}=0, \\
\left(\phi_{0}\right)^{a b} \phi_{b}{ }^{i}=0 .
\end{gathered}
$$

The condition (2.13) gives $\phi_{0}=0$ or $\phi=0$. Since the latter solution is inconsistent with (2.9), we have $\phi_{0}=0$. The scalar field $\Sigma$ can be diagonalized by a $U(N)$ gauge transformation as

$$
\Sigma=\operatorname{diag}\left(\Sigma_{1}, \Sigma_{2}, \ldots, \Sigma_{N}\right) .
$$

Since $M$ and $\Sigma$ in (2.12) are both diagonal matrices, the vacuum solutions to (2.12) are labeled by

$$
\left(\Sigma_{1}, \Sigma_{2}, \ldots, \Sigma_{N}\right)=\left( \pm m_{1}, \pm m_{2}, \ldots, \pm m_{N}\right) .
$$

There exist $2^{N-1}$ vacua in the nonlinear sigma model on $S O(2 N) / U(N)$ since the tensor (2.2) is invariant under $O(2 N)$, which includes a parity transformation. On the other hand, there exist $2^{N}$ vacua in the nonlinear sigma model on $S p(N) / U(N)$. The numbers are the Euler characteristics of the spaces [20].

To study wall solutions, we assume that fields are static and all the fields depend only on the $x_{1} \equiv x$ coordinate. We also assume that there is Poincare invariance on the two-dimensional world volume of walls so we can set $A_{0}=A_{2}=0$. The energy density along the $x$ direction is

$$
\begin{aligned}
\mathcal{E} & =\left(\left|(D \phi)_{a}{ }^{i}\right|^{2}+\left|\phi_{a}{ }^{j} M_{j}{ }^{i}-\Sigma_{a}{ }^{b} \phi_{b}{ }^{i}\right|^{2}+4\left|\left(\phi_{0}\right)^{a b} \phi_{b}{ }^{i}\right|^{2}\right) \\
& =\left(\left|(D \phi)_{a}{ }^{i} \mp\left(\phi_{a}{ }^{j} M_{j}{ }^{i}-\Sigma_{a}{ }^{b} \phi_{b}{ }^{i}\right)\right|^{2}+4\left|\left(\phi_{0}\right)^{a b} \phi_{b}{ }^{i}\right|^{2}\right) \pm \mathcal{T} \\
& \geq \pm \mathcal{T},
\end{aligned}
$$

with $D \equiv D_{\mu=1}$ and

$$
\mathcal{T}=\partial\left(\phi_{a}{ }^{i} M_{i}{ }^{j} \bar{\phi}_{j}{ }^{a}\right), \quad \partial \equiv \partial_{1},
$$

which is the tension density of the wall. The tension is

$$
T=\int_{-\infty}^{+\infty} d x \partial \operatorname{Tr}(\phi M \bar{\phi})=[\operatorname{Tr}(\phi M \bar{\phi})]_{-\infty}^{+\infty} .
$$

The energy density is constrained by (2.9) and (2.10).

We choose the upper sign for the Bogomol'nyi-PrasadSommerfield (BPS) equation and the lower sign for the anti-BPS equation in the first squared term in (2.16). Then, the BPS equation is

$$
(D \phi)_{a}{ }^{i}-\left(\phi_{a}{ }^{j} M_{j}{ }^{i}-\Sigma_{a}{ }^{b} \phi_{b}{ }^{i}\right)=0 .
$$

We introduce complex matrix functions $S_{a}{ }^{b}(x)$ and $f_{a}{ }^{i}(x)$, which are defined by

$\Sigma_{a}{ }^{b}-i A_{a}{ }^{b}=\left(S^{-1} \partial S\right)_{a}{ }^{b}, \quad \phi_{a}{ }^{i}=\left(S^{-1}\right)_{a}{ }^{b} f_{b}{ }^{i}$.

Then, the equation (2.19) is solved by

$$
f_{b}{ }^{i}=H_{0 b}{ }^{j}\left(e^{M x}\right)_{j}{ }^{i} .
$$

Therefore, the solution to the BPS equation (2.19) is

$$
\phi_{a}{ }^{i}=\left(S^{-1}\right)_{a}{ }^{b} H_{0 b}{ }^{j}\left(e^{M x}\right)_{j}{ }^{i} .
$$

The coefficient matrix $H_{0}$ is the moduli matrix. $\Sigma, A$, and $\phi$ are invariant under the transformations

$$
S^{\prime}{ }_{a}^{b}=V_{a}{ }^{c} S_{c}{ }^{b}, \quad H^{\prime}{ }^{i}{ }^{i}=V_{a}{ }^{c} H_{0 c}{ }^{i},
$$

where $V \in G L(N, \mathbf{C})$. The matrix $V$ defines an equivalent class of $\left(S, H_{0}\right)$. This is named the world volume symmetry in the moduli matrix formalism $[8,9]$. Equations $(2.9)$ and (2.10) correspond to

$$
H_{0 a}{ }^{i}\left(e^{2 M x}\right)_{i}{ }^{j} H_{0 j}^{\dagger} b=\left(S S^{\dagger}\right)_{a}{ }^{b} \equiv \Omega_{a}{ }^{b},
$$

$H_{0 a}{ }^{i} J_{i j} H^{T j}{ }_{b}=0, \quad($ Hermitian conjugate $)=0$.

From (2.23) and (2.25), we can learn that moduli matrices $H_{0}$ 's parametrize $S p(N) / U(N)$. The tension density (2.17) is

$$
\mathcal{T}=\frac{1}{2} \partial^{2} \ln \operatorname{det} \Omega
$$

In the moduli matrix formalism, walls are constructed from elementary walls. The elementary wall operators are 
the simple root generators of the flavor symmetry. So, the elementary walls can be identified with the simple roots [17]. We summarize the simple root generators $E_{i},(i=$ $1, \ldots, N)$ and the simple roots $\vec{\alpha}_{i}$ of $S O(2 N)$ and $U S p(2 N)$ following the convention of Refs. [15,16]. The set of vectors $\left\{\hat{e}_{i}\right\}$ is the standard unit vectors $\hat{e}_{i} \cdot \hat{e}_{j}=\delta_{i j}$ :

(i) $S O(2 N)$ :

$$
\begin{aligned}
E_{i} & =e_{i, i+1}-e_{i+N+1, i+N}, \quad(i=1, \ldots, N-1), \\
E_{N} & =e_{N-1,2 N}-e_{N, 2 N-1}, \\
\vec{\alpha}_{i} & =\hat{e}_{i}-\hat{e}_{i+1}, \\
\vec{\alpha}_{N} & =\hat{e}_{N-1}+\hat{e}_{N} .
\end{aligned}
$$

(ii) $\operatorname{USp}(2 N)$ :

$$
\begin{aligned}
E_{i} & =e_{i, i+1}-e_{i+N+1, i+N}, \quad(i=1, \ldots, N-1), \\
E_{N} & =e_{N, 2 N}, \\
\vec{\alpha}_{i} & =\hat{e}_{i}-\hat{e}_{i+1}, \\
\vec{\alpha}_{N} & =2 \hat{e}_{N} .
\end{aligned}
$$

The Cartan generators (2.5) and the root generators (2.28) are related and normalized by

$$
\begin{aligned}
\operatorname{Tr}\left(H_{I} H_{J}\right) & =2 \delta_{I J}, \quad(I, J=1, \ldots, N), \\
\operatorname{Tr}\left(H_{I} E_{i}\right) & =0, \\
\operatorname{Tr}\left(E_{i} E_{i}^{\dagger}\right) & =\frac{4}{\vec{\alpha}_{i} \cdot \vec{\alpha}_{i}} .
\end{aligned}
$$

In this paper, $\langle A\rangle$ denotes a vacuum, and $\langle A \leftarrow B\rangle$ denotes a wall that connects vacuum $\langle A\rangle$ and vacuum $\langle B\rangle$.

The mass matrix $M$ (2.6), which is a linear combination of the Cartan generators, and elementary wall $\langle A \leftarrow B\rangle$, which is generated by Cartan generator $E_{i}$, are related by

$$
c\left[M, E_{i}\right]=c\left(\vec{m} \cdot \vec{\alpha}_{i}\right) E_{i}=T_{\langle A \leftarrow B\rangle} E_{i},
$$

where $c$ is a constant and $T_{\langle A \leftarrow B\rangle}$ is the tension of wall. The moduli matrix of elementary wall $H_{0\langle A \leftarrow B\rangle}$, which connects $\langle A\rangle$ and $\langle B\rangle$, is

$$
\begin{gathered}
H_{0\langle A \leftarrow B\rangle}=H_{0\langle A\rangle} e^{E_{i}(r)}, \\
E_{i}(r) \equiv e^{r} E_{i}, \quad(i=1, \ldots, N),
\end{gathered}
$$

where $E_{i}$ is an elementary wall operator and $r$ is a complex parameter with $-\infty<\operatorname{Re}(r)<+\infty$.

Unlike $S U(N)$ and $S O(2 N)$, the lengths of the simple roots of $U S p(2 N)$ are different. Therefore, the constant $c$ in (2.30) can be different in some vacuum sectors of the nonlinear sigma models on $\operatorname{Sp}(N) / U(N)$.

We first review the formalism for the walls of the nonlinear sigma models on $G_{N_{F}, N_{C}}$ and $S O(2 N) / U(N)$. In this case, $c$ is the same in all the sectors of the vacuum structure. Given the aim of the work [11], it can be fixed as $c=1$ for convenience. Elementary walls can be compressed to single walls. In the nonlinear sigma models on $G_{N_{F}, N_{C}}$ and on $S O(2 N) / U(N)$, a compressed wall of level $n$ that connects $\langle A\rangle$ and $\left\langle A^{\prime}\right\rangle$ is

$$
\begin{aligned}
H_{0\left\langle A \leftarrow A^{\prime}\right\rangle}= & H_{0\langle A\rangle} e^{\left.\left[E_{i_{1}},\left[E_{i_{2}},\left[E_{i_{3}},\left[\cdots,\left[E_{i_{n}}, E_{i_{n+1}}\right]\right]\right]\right] \cdots\right]\right](r)}, \\
& \left(i_{m}=1, \ldots, N ; m=1, \ldots, n+1\right) .
\end{aligned}
$$

A double-wall moduli matrix is constructed by multiplying a single-wall operator to a single-wall moduli matrix. By repeating it, we get a triple wall, a quadruple wall, and so on. A multiwall, which interpolates $\langle A\rangle,\left\langle A^{\prime}\right\rangle, \cdots$, and $\langle B\rangle$, is

$$
\begin{array}{r}
H_{0\left\langle A \leftarrow A^{\prime} \leftarrow \cdots \leftarrow B\right\rangle}=H_{0\langle A\rangle} e^{E_{i_{1}}\left(r_{1}\right)} e^{E_{i_{2}}\left(r_{2}\right)} \cdots e^{E_{i_{n}}\left(r_{n}\right)}, \\
\left(i_{m}=1, \ldots, N ; m=1, \ldots, n\right),
\end{array}
$$

where parameters $r_{i}(i=1,2, \ldots)$ are complex parameters ranging $-\infty<\operatorname{Re}\left(r_{i}\right)<\infty$. Elementary walls pass through each other if

$$
\left[E_{i_{m}}, E_{i_{n}}\right]=0,
$$

and these walls are named penetrable walls [9].

Elementary walls can be identified with simple roots by (2.30) [17]. Let root vector $\vec{g}_{\left\langle A_{1} \leftarrow A_{2}\right\rangle}$ denote the wall that connects vacuum $\left\langle A_{1}\right\rangle$ and vacuum $\left\langle A_{2}\right\rangle$. The corresponding tension of the wall is $T_{\left\langle A_{1} \leftarrow A_{2}\right\rangle}=\vec{m} \cdot \vec{g}_{\left\langle A_{1} \leftarrow A_{2}\right\rangle}$. Then, the elementary wall of (2.31) is

$$
\vec{g}_{\langle A \leftarrow B\rangle} \equiv c \vec{\alpha}_{i} .
$$

The compressed wall of (2.32) is

$\vec{g}_{\left\langle A \leftarrow A^{\prime}\right\rangle} \equiv c \vec{\alpha}_{i_{1}}+c \vec{\alpha}_{i_{2}}+c \vec{\alpha}_{i_{3}}+\cdots c \vec{\alpha}_{i_{n}}+c \vec{\alpha}_{i_{n+1}}$.

The root vectors of the two penetrable elementary walls of (2.34) are orthogonal,

$$
\vec{\alpha}_{i_{m}} \cdot \vec{\alpha}_{i_{n}}=0
$$

Now, we study walls of the nonlinear sigma models on $S p(N) / U(N)$. In this case, $c=2$ for $i=1, \ldots, N-1$, and $c=1$ for $i=N$ in (2.30). An elementary wall $\left\langle A \leftarrow B^{\prime}\right\rangle$ is

$$
\vec{g}_{\left\langle A \leftarrow B^{\prime}\right\rangle}=c \vec{\alpha}_{i} .
$$

The moduli matrix of $\left\langle A \leftarrow A^{\prime \prime}\right\rangle$, which is a compressed wall of level $n$, is

$$
\begin{aligned}
H_{0\left\langle A \leftarrow A^{\prime \prime}\right\rangle} & =H_{0\langle A\rangle} e^{\left[E_{i_{1}},\left[E_{i_{2}},\left[E_{i_{3}},\left[\cdots,\left[E_{i_{n}}, E_{i_{n+1}}\right]\right]\right] \cdots\right]\right](r)}, \\
& \left(i_{m}=1, \ldots, N-1 ; m=1, \ldots, n+1\right) .
\end{aligned}
$$

The moduli matrices and the operators are the same as (2.32) for $i=1, \ldots, N-1$. However, the formula should change for operator $E_{N}$. As an example, an elementary wall 
$H_{0\left\langle B \leftarrow B^{\prime}\right\rangle}=H_{0\langle B\rangle} e^{E_{N-1}(r)} \quad$ and an elementary wall $H_{0\left\langle B^{\prime} \leftarrow B^{\prime \prime}\right\rangle}=H_{0\left\langle B^{\prime}\right\rangle} e^{E_{N}(r)}$ are compressed to

$$
H_{0\left\langle B \leftarrow B^{\prime \prime}\right\rangle}=H_{0\langle B\rangle} e^{\left[E_{N-1},\left[E_{N-1}, E_{N}\right]\right](r)}
$$

or

$$
H_{0\left\langle B \leftarrow B^{\prime \prime}\right\rangle}=H_{0\langle B\rangle} e^{\left[\left[E_{N}, E_{N-1}\right], E_{N-1}\right](r)} .
$$

The formulas for multiwalls (2.33) and for penetrable walls (2.34) hold for walls of nonlinear sigma models on $\operatorname{Sp}(N) / U(N)$.

The compressed wall of (2.39) in terms of root vectors is

$\vec{g}_{\left\langle A \leftarrow A^{\prime \prime}\right\rangle}=2 \vec{\alpha}_{i_{1}}+2 \vec{\alpha}_{i_{2}}+2 \vec{\alpha}_{i_{3}}+\cdots 2 \vec{\alpha}_{i_{n}}+2 \vec{\alpha}_{i_{n+1}}$,

whereas the compressed wall of (2.40) and (2.41) is

$$
\vec{g}_{\left\langle B \leftarrow B^{\prime \prime}\right\rangle}=2 \vec{\alpha}_{N-1}+\vec{\alpha}_{N} .
$$

In this paper, we label the moduli matrices of vacua in descending order as

$$
\begin{aligned}
\left(\Sigma_{1}, \Sigma_{2}, \ldots, \Sigma_{N-1}, \Sigma_{N}\right) & =\left(m_{1}, m_{2}, \ldots, m_{N-1}, m_{N}\right), \\
\left(\Sigma_{1}, \Sigma_{2}, \ldots, \Sigma_{N-1}, \Sigma_{N}\right) & =\left(m_{1}, m_{2}, \ldots, m_{N-1},-m_{N}\right), \\
\left(\Sigma_{1}, \Sigma_{2}, \ldots, \Sigma_{N-1}, \Sigma_{N}\right) & =\left(m_{1}, m_{2}, \ldots,-m_{N-1}, m_{N}\right), \\
\left(\Sigma_{1}, \Sigma_{2}, \ldots, \Sigma_{N-1}, \Sigma_{N}\right) & =\left(m_{1}, m_{2}, \ldots,-m_{N-1},-m_{N}\right), \\
& \vdots \\
\left(\Sigma_{1}, \Sigma_{2}, \ldots, \Sigma_{N-1}, \Sigma_{N}\right) & =\left(m_{1},-m_{2}, \ldots,-m_{N-1},-m_{N}\right), \\
\left(\Sigma_{1}, \Sigma_{2}, \ldots, \Sigma_{N-1}, \Sigma_{N}\right) & =\left(-m_{1}, m_{2}, \ldots, m_{N-1}, m_{N}\right), \\
& \vdots \\
\left(\Sigma_{1}, \Sigma_{2}, \ldots, \Sigma_{N-1}, \Sigma_{N}\right) & =\left(-m_{1},-m_{2}, \ldots,-m_{N-1},-m_{N}\right) .
\end{aligned}
$$

\section{NONLINEAR SIGMA MODELS ON $\operatorname{Sp}(\boldsymbol{N}) / \boldsymbol{U}(\boldsymbol{N})$ WITH $N \leq 6$}

There are two vacua in the nonlinear sigma model on $S p(1) / U(1)$,

$$
\begin{array}{ll}
\Phi_{\langle 1\rangle}=(1,0), & \Sigma=m, \\
\Phi_{\langle 2\rangle}=(0,1), & \Sigma=-m .
\end{array}
$$

The moduli matrices of the vacua are

$$
\begin{array}{ll}
H_{0\langle 1\rangle}=(1,0), & \Sigma=m, \\
H_{0\langle 2\rangle}=(0,1), & \Sigma=-m .
\end{array}
$$

There is only one wall, which is an elementary wall. The elementary wall operator is

$$
E_{1}=e_{1,2}
$$

and the moduli matrix of the elementary wall is

$$
H_{0\langle 1 \leftarrow 2\rangle}=H_{0\langle 1\rangle} e^{E(r)}=\left(1, e^{r}\right) .
$$

The tension of the wall is

$$
T_{\langle 1 \leftarrow 2\rangle}=\vec{m} \cdot \vec{\alpha}_{1} .
$$

The diagram of the elementary wall is depicted in Fig. 1(a).

We study walls of the nonlinear sigma model on $S p(2) / U(2)$. The Cartan generators $H_{I},(I=1,2)$, the simple root generators $E_{i},(i=1,2)$, and the simple roots of $U S p(4)$ are

$$
\begin{aligned}
H_{1} & =e_{1,1}-e_{3,3}, \quad H_{2}=e_{2,2}-e_{4,4}, \\
E_{1} & =e_{1,2}-e_{4,3}, \quad E_{2}=e_{2,4}, \\
\vec{\alpha}_{1} & =\hat{e}_{1}-\hat{e}_{2}, \quad \vec{\alpha}_{2}=2 \hat{e}_{2} .
\end{aligned}
$$

For $N=2$, the vacuum condition (2.12) gives rise to four vacua, which have the following form:

$$
\begin{aligned}
& \Phi_{\langle 1\rangle}=\left(\begin{array}{llll}
1 & 0 & 0 & 0 \\
0 & 1 & 0 & 0
\end{array}\right), \quad\left(\Sigma_{1}, \Sigma_{2}\right)=\left(m_{1}, m_{2}\right), \\
& \Phi_{\langle 2\rangle}=\left(\begin{array}{llll}
1 & 0 & 0 & 0 \\
0 & 0 & 0 & 1
\end{array}\right), \quad\left(\Sigma_{1}, \Sigma_{2}\right)=\left(m_{1},-m_{2}\right), \\
& \Phi_{\langle 3\rangle}=\left(\begin{array}{llll}
0 & 0 & 1 & 0 \\
0 & 1 & 0 & 0
\end{array}\right), \quad\left(\Sigma_{1}, \Sigma_{2}\right)=\left(-m_{1}, m_{2}\right), \\
& \Phi_{\langle 4\rangle}=\left(\begin{array}{llll}
0 & 0 & 1 & 0 \\
0 & 0 & 0 & 1
\end{array}\right), \quad\left(\Sigma_{1}, \Sigma_{2}\right)=\left(-m_{1},-m_{2}\right) .
\end{aligned}
$$

The moduli matrices of (3.7) are

$$
\begin{aligned}
& H_{0\langle 1\rangle}=\left(\begin{array}{llll}
1 & 0 & 0 & 0 \\
0 & 1 & 0 & 0
\end{array}\right), \quad\left(\Sigma_{1}, \Sigma_{2}\right)=\left(m_{1}, m_{2}\right), \\
& H_{0\langle 2\rangle}=\left(\begin{array}{llll}
1 & 0 & 0 & 0 \\
0 & 0 & 0 & 1
\end{array}\right), \quad\left(\Sigma_{1}, \Sigma_{2}\right)=\left(m_{1},-m_{2}\right), \\
& H_{0\langle 3\rangle}=\left(\begin{array}{llll}
0 & 0 & 1 & 0 \\
0 & 1 & 0 & 0
\end{array}\right), \quad\left(\Sigma_{1}, \Sigma_{2}\right)=\left(-m_{1}, m_{2}\right), \\
& H_{0\langle 4\rangle}=\left(\begin{array}{llll}
0 & 0 & 1 & 0 \\
0 & 0 & 0 & 1
\end{array}\right), \quad\left(\Sigma_{1}, \Sigma_{2}\right)=\left(-m_{1},-m_{2}\right) .
\end{aligned}
$$

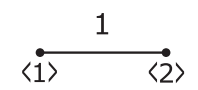

(a)

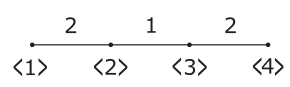

(b)

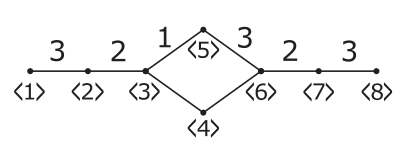

(c)

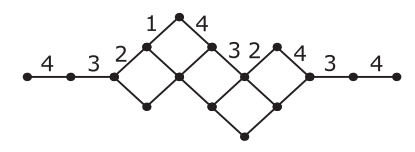

(d)
FIG. 1. Elementary walls of the nonlinear sigma models on $S p(N) / U(N)$. (a) $N=1$, (b) $N=2$, (c) $N=3$, and (d) $N=4$. The numbers indicate the subscript $i$ 's of roots $\vec{\alpha}_{i}$. The left-hand side is the limit as $x \rightarrow+\infty$, and the right-hand side is the limit as $x \rightarrow-\infty$. 
The moduli matrices of elementary walls that connect the vacua (3.8) are

$$
\begin{aligned}
& H_{\langle 1 \leftarrow 2\rangle}=H_{\langle 1\rangle} e^{E_{2}(r)}=\left(\begin{array}{cccc}
1 & 0 & 0 & 0 \\
0 & 1 & 0 & e^{r}
\end{array}\right), \\
& H_{\langle 2 \leftarrow 3\rangle}=H_{\langle 2\rangle} e^{E_{1}(r)}=\left(\begin{array}{cccc}
1 & e^{r} & 0 & 0 \\
0 & 0 & -e^{r} & 1
\end{array}\right), \\
& H_{\langle 3 \leftarrow 4\rangle}=H_{\langle 3\rangle} e^{E_{2}(r)}=\left(\begin{array}{cccc}
0 & 0 & 1 & 0 \\
0 & 1 & 0 & e^{r}
\end{array}\right) .
\end{aligned}
$$

$$
\begin{aligned}
\phi_{23} & =\left(\begin{array}{cccc}
e^{m_{1} x} \Delta_{1}^{-1 / 2} & e^{m_{2} x+r} \Delta_{1}^{-1 / 2} & 0 & 0 \\
0 & 0 & -e^{-m_{1} x+r} \Delta_{2}^{-1 / 2} & e^{-m_{2} x} \Delta_{2}^{-1 / 2}
\end{array}\right), \\
\Delta_{1} & =e^{2 m_{1} x}+e^{2 m_{2} x+2 \operatorname{Re}(r)} \\
\Delta_{2} & =e^{-2 m_{1} x+2 \operatorname{Re}(r)}+e^{-2 m_{2} x} .
\end{aligned}
$$

The wall (3.12) has the limits

$$
\begin{aligned}
x \rightarrow+\infty, & \phi_{23} & \rightarrow \Phi_{\langle 2\rangle}, \\
x \rightarrow-\infty, & \phi_{23} & \rightarrow\left(\begin{array}{llll}
0 & 1 & 0 & 0 \\
0 & 0 & 1 & 0
\end{array}\right) .
\end{aligned}
$$

Here, $\phi_{23}(x \rightarrow-\infty)$ is related to vacuum $\Phi_{3}$ by a $U(N)$ gauge transformation. Therefore, $\phi_{23}(x \rightarrow-\infty)$ and $\Phi_{3}$ are the same vacuum. We can also see this by making use of the world volume symmetry. The moduli matrix of $\phi_{23}(x \rightarrow$ $-\infty)$ is

$$
H_{0\langle 3\rangle}^{\prime}=\left(\begin{array}{cccc}
0 & 1 & 0 & 0 \\
0 & 0 & 1 & 0
\end{array}\right),
$$

which is related to $H_{0\langle 3\rangle}$ by the world volume symmetry

$$
H_{0\langle 3\rangle}^{\prime}=\left(\begin{array}{cc}
0 & 1 \\
1 & 0
\end{array}\right) H_{0\langle 3\rangle} .
$$

Therefore, Eq. (3.12) is the elementary wall that connects vacuum $\langle 2\rangle$ and vacuum $\langle 3\rangle$. The wall solution (2.22) with $H_{\langle 3 \leftarrow 4\rangle}$ is

$$
\begin{aligned}
\phi_{34} & =\left(\begin{array}{cccc}
0 & 0 & 1 & 0 \\
0 & e^{m_{2} x} \Delta^{-1 / 2} & 0 & e^{-m_{2} x+r} \Delta^{-1 / 2}
\end{array}\right), \\
\Delta & =e^{2 m_{2} x}+e^{-2 m_{2} x+2 \operatorname{Re}(r)} .
\end{aligned}
$$

The wall solution (3.16) has the limits
The wall solution (2.22) with $H_{\langle 1 \leftarrow 2\rangle}$ is

$$
\begin{aligned}
\phi_{12} & =\left(\begin{array}{cccc}
1 & 0 & 0 & 0 \\
0 & e^{m_{2} x} \Delta^{-1 / 2} & 0 & e^{-m_{2} x+r} \Delta^{-1 / 2}
\end{array}\right), \\
\Delta & =e^{2 m_{2} x}+e^{-2 m_{2} x+2 \operatorname{Re}(r)} .
\end{aligned}
$$

All the phases, which appear due to the $U(1)$ gauge symmetry, are fixed to zero. The wall (3.10) has the limits

$$
\begin{array}{llrl}
x & \rightarrow+\infty, & \phi_{12} & \rightarrow \Phi_{\langle 1\rangle}, \\
x & \rightarrow-\infty, & \phi_{12} & \rightarrow \Phi_{\langle 2\rangle},
\end{array}
$$

as expected. The wall solution (2.22) with $H_{\langle 2 \leftarrow 3\rangle}$ is

$$
\begin{aligned}
x & \rightarrow+\infty, & \phi_{34} & \rightarrow \Phi_{\langle 3\rangle}, \\
x & \rightarrow-\infty, & \phi_{34} & \rightarrow \Phi_{\langle 4\rangle} .
\end{aligned}
$$

Tension $T_{\langle A \leftarrow B\rangle}$ of the wall that connects vacuum $\Phi_{\langle A\rangle}$ and vacuum $\Phi_{\langle B\rangle}$ is obtained from (3.7). The tensions of the elementary walls are

$$
\begin{gathered}
T_{\langle 1 \leftarrow 2\rangle}=\vec{m} \cdot \vec{\alpha}_{2}, \\
T_{\langle 2 \leftarrow 3\rangle}=2 \vec{m} \cdot \vec{\alpha}_{1}, \\
T_{\langle 3 \leftarrow 4\rangle}=\vec{m} \cdot \vec{\alpha}_{2} .
\end{gathered}
$$

Therefore, the elementary walls are identified with

$$
\begin{aligned}
& \vec{g}_{\langle 1 \leftarrow 2\rangle}=\vec{g}_{\langle 3 \leftarrow 4\rangle}=\vec{\alpha}_{2}, \\
& \vec{g}_{\langle 2 \leftarrow 3\rangle}=2 \vec{\alpha}_{1} .
\end{aligned}
$$

The diagram of the elementary walls are depicted in Fig. 1(b). We omit the coefficients of the simple roots in elementary wall diagrams in this paper. From the diagram in Fig. 1(b), one can see how a compressed walls is constructed. From (2.39), the compressed wall that interpolates $\langle 1\rangle$ and $\langle 3\rangle$ is

$$
H_{0\langle 1 \leftarrow 3\rangle}=H_{0\langle 1\rangle} e^{\left[\left[E_{2}, E_{1}\right], E_{1}\right](r)}=\left(\begin{array}{cccc}
1 & 0 & 2 e^{r} & 0 \\
0 & 1 & 0 & 0
\end{array}\right),
$$

and the compressed wall that interpolates $\langle 2\rangle$ and $\langle 4\rangle$ is 


$$
H_{0\langle 2 \leftarrow 4\rangle}=H_{0\langle 2\rangle} e^{\left[E_{1},\left[E_{1}, E_{2}\right]\right](r)}=\left(\begin{array}{cccc}
1 & 0 & 2 e^{r} & 0 \\
0 & 0 & 0 & 1
\end{array}\right) .
$$

These are the compressed walls of level 1 .

It can be shown that these compressed walls can be obtained from double walls. Moduli matrices of double walls $\langle 1 \leftarrow 2 \leftarrow 3\rangle$ and $\langle 2 \leftarrow 3 \leftarrow 4\rangle$ are

$$
\begin{aligned}
& H_{0\langle 1 \leftarrow 2 \leftarrow 3\rangle}=H_{0\langle 1 \leftarrow 2\rangle} e^{E_{1}\left(r_{2}\right)}=\left(\begin{array}{cccc}
1 & e^{r_{2}} & 0 & 0 \\
0 & 1 & -e^{r_{1}+r_{2}} & e^{r_{1}}
\end{array}\right), \\
& H_{0\langle 2 \leftarrow 3 \leftarrow 4\rangle}=H_{0\langle 2 \leftarrow 3\rangle} e^{E_{2}\left(r_{2}\right)}=\left(\begin{array}{cccc}
1 & e^{r_{1}} & 0 & e^{r_{1}+r_{2}} \\
0 & 0 & -e^{r_{1}} & 1
\end{array}\right) .
\end{aligned}
$$

$H_{0\langle 1 \leftarrow 2 \leftarrow 3\rangle}$ can be transformed as

$$
\begin{aligned}
H_{0\langle 1 \leftarrow 2 \leftarrow 3\rangle} & \rightarrow\left(\begin{array}{cc}
1 & -e^{r_{2}} \\
e^{-2 r_{2}} & 1
\end{array}\right)\left(\begin{array}{cccc}
1 & e^{r_{2}} & 0 & 0 \\
0 & 1 & -e^{r_{1}+r_{2}} & e^{r_{1}}
\end{array}\right) \\
& =\left(\begin{array}{cccc}
1 & 0 & e^{r+\ln 2} & -e^{r-r_{2}+\ln 2} \\
e^{-2 r_{2}} & 1+e^{-r_{2}} & -e^{r-r_{2}+\ln 2} & e^{r-2 r_{2}+\ln 2}
\end{array}\right),
\end{aligned}
$$

where $r:=r_{1}+2 r_{2}-\ln 2$. The limit of $H_{0\langle 1 \leftarrow 2 \leftarrow 3\rangle}$ in (3.25) as $r_{2} \rightarrow+\infty$ with finite $r$ equals $H_{0\langle 1 \leftarrow 3\rangle}$ in (3.22), or equivalently,

$$
\begin{aligned}
H_{0\langle 1 \leftarrow 2 \leftarrow 3\rangle} & =H_{0\langle 1\rangle} e^{E_{2}\left(r_{1}\right)} e^{E_{1}\left(r_{2}\right)} \\
& \simeq H_{0\langle 1\rangle} e^{E_{2}\left(r_{1}\right)} e^{\left[E_{1}, E_{2}\right]\left(r_{1}+r_{2}+i \pi\right)} e^{\left[E_{1},\left[E_{1}, E_{2}\right]\right]\left(r_{1}+2 r_{2}-\ln 2\right)} \\
= & H_{0\langle 1\rangle} e^{E_{2}\left(r-2 r_{2}+\ln 2\right)} e^{\left[E_{1}, E_{2}\right]\left(r-r_{2}+\ln 2+i \pi\right)} \\
& \times e^{\left[E_{1},\left[E_{1}, E_{2}\right]\right](r)}
\end{aligned}
$$

where $r:=r_{1}+2 r_{2}-\ln 2$ and $\simeq$ means the following world volume symmetry transformation:

$$
H_{0\langle 1\rangle} e^{E_{1}\left(r_{2}\right)}=\left(\begin{array}{cc}
1 & e^{r_{2}} \\
0 & 1
\end{array}\right) H_{0\langle 1\rangle} \simeq H_{0\langle 1\rangle} .
$$

As $r_{2} \rightarrow+\infty$ with finite $r, H_{0\langle 1 \leftarrow 2 \leftarrow 3\rangle} \rightarrow H_{0\langle 1 \leftarrow 3\rangle}$.

$$
H_{0\langle 2 \leftarrow 3 \leftarrow 4\rangle} \text { transforms as }
$$

$$
\begin{aligned}
H_{0\langle 2 \leftarrow 3 \leftarrow 4\rangle} & \rightarrow\left(\begin{array}{cc}
1 & -e^{r_{1}+r_{2}} \\
0 & 1
\end{array}\right)\left(\begin{array}{cccc}
1 & e^{r_{1}} & 0 & e^{r_{1}+r_{2}} \\
0 & 0 & -e^{r_{1}} & 1
\end{array}\right) \\
& =\left(\begin{array}{cccc}
1 & e^{r_{1}} & e^{r+\ln 2} & 0 \\
0 & 0 & -e^{r_{1}} & 1
\end{array}\right),
\end{aligned}
$$

where $r:=2 r_{1}+r_{2}-\ln 2$. The limit of $H_{0\langle 2 \leftarrow 3 \leftarrow 4\rangle}$ in (3.28) as $r_{1} \rightarrow-\infty$ with finite $r$ equals $H_{0\langle 2 \leftarrow 4\rangle}$ in (3.23), or equivalently,

$$
\begin{aligned}
H_{0\langle 2 \leftarrow 3 \leftarrow 4\rangle}= & H_{0\langle 2\rangle} e^{E_{1}\left(r_{1}\right)} e^{E_{2}\left(r_{2}\right)} \\
= & H_{0\langle 2\rangle} e^{E_{2}\left(r_{2}\right)} e^{E_{1}\left(r_{1}\right)} e^{\left[E_{1}, E_{2}\right]\left(r_{1}+r_{2}\right)} \\
& \times e^{\left[E_{1},\left[E_{1}, E_{2}\right]\right]\left(2 r_{1}+r_{2}-\ln 2+i \pi\right)} \\
= & H_{0\langle 2\rangle} e^{\left[E_{1}, E_{2}\right]\left(r_{1}+r_{2}\right)} e^{E_{1}\left(r_{1}\right)+\left[E_{1},\left[E_{1}, E_{2}\right]\right]\left(2 r_{1}+r_{2}\right)} \\
& \times e^{\left[E_{1},\left[E_{1}, E_{2}\right]\right]\left(2 r_{1}+r_{2}-\ln 2+i \pi\right)} \\
\simeq & H_{0\langle 2\rangle} e^{E_{1}\left(r_{1}\right)+\left[E_{1},\left[E_{1}, E_{2}\right]\right]\left(2 r_{1}+r_{2}\right)} \\
& \times e^{\left[E_{1},\left[E_{1}, E_{2}\right]\right]\left(2 r_{1}+r_{2}-\ln 2+i \pi\right)},
\end{aligned}
$$

where $r:=2 r_{1}+r_{2}-\ln 2$ and $\simeq$ means the following world volume symmetry transformation:

$H_{0\langle 2\rangle} e^{\left[E_{1}, E_{2}\right]\left(r_{1}+r_{2}\right)}=\left(\begin{array}{cc}1 & e^{r_{1}+r_{2}} \\ 0 & 1\end{array}\right) H_{0\langle 2\rangle} \simeq H_{0\langle 2\rangle}$.

As $r_{1} \rightarrow-\infty$ with finite $r, H_{0\langle 2 \leftarrow 3 \leftarrow 4\rangle} \rightarrow H_{0\langle 2 \leftarrow 4\rangle}$.

The triple wall $H_{0\langle 1 \leftarrow 2 \leftarrow 3 \leftarrow 4\rangle}$ is

$$
\begin{aligned}
H_{0\langle 1 \leftarrow 2 \leftarrow 3 \leftarrow 4\rangle} & =H_{0\langle 1 \leftarrow 2 \leftarrow 3\rangle} e^{E_{2}\left(r_{3}\right)} \\
& =\left(\begin{array}{cccc}
1 & e^{r_{2}} & 0 & e^{r_{2}+r_{3}} \\
0 & 1 & -e^{r_{1}+r_{2}} & e^{r_{1}}+e^{r_{3}}
\end{array}\right),
\end{aligned}
$$

which consists of three elementary walls $\langle 1 \leftarrow 2\rangle,\langle 2 \leftarrow 3\rangle$, and $\langle 3 \leftarrow 4\rangle$. Since $\left[E_{2},\left[E_{1},\left[E_{1}, E_{2}\right]\right]\right]=0$ or equivalently $\vec{\alpha}_{2} \cdot\left(2 \vec{\alpha}_{1}+\vec{\alpha}_{2}\right)=0$, the triple wall $\langle 1 \leftarrow 2 \leftarrow 3 \leftarrow 4\rangle$ cannot be compressed to a compressed wall of level 2 . Instead, the elementary wall $\langle 1 \leftarrow 2\rangle$ and compressed wall $\langle 2 \leftarrow 4\rangle$ are penetrable each other. Compressed wall $\langle 1 \leftarrow 3\rangle$ and elementary wall $\langle 3 \leftarrow 4\rangle$ are also penetrable each other. $\langle 2 \leftarrow 4\rangle$ and $\langle 1 \leftarrow 3\rangle$ are compressed walls of level 1.

We study walls of the nonlinear sigma model on $S p(3) / U(3)$. The simple root generators and the simple roots of $U S p(6)$ are

$$
\begin{aligned}
& E_{1}=e_{1,2}-e_{5,4}, \\
& E_{2}=e_{2,3}-e_{6,5}, \\
& E_{3}=e_{3,6},
\end{aligned}
$$

and

$$
\begin{aligned}
& \vec{\alpha}_{1}=\hat{e}_{1}-\hat{e}_{2}, \\
& \vec{\alpha}_{2}=\hat{e}_{2}-\hat{e}_{3}, \\
& \vec{\alpha}_{3}=2 \hat{e}_{3} .
\end{aligned}
$$

The eight vacua of the nonlinear sigma model on $S p(3) / U(3)$ are labeled in the descending order of (2.44): 


$$
\begin{aligned}
& \langle 1\rangle:\left(\Sigma_{1}, \Sigma_{2}, \Sigma_{3}\right)=\left(m_{1}, m_{2}, m_{3}\right), \\
& \langle 2\rangle:\left(\Sigma_{1}, \Sigma_{2}, \Sigma_{3}\right)=\left(m_{1}, m_{2},-m_{3}\right), \\
& \langle 3\rangle:\left(\Sigma_{1}, \Sigma_{2}, \Sigma_{3}\right)=\left(m_{1},-m_{2}, m_{3}\right), \\
& \langle 4\rangle:\left(\Sigma_{1}, \Sigma_{2}, \Sigma_{3}\right)=\left(m_{1},-m_{2},-m_{3}\right), \\
& \langle 5\rangle:\left(\Sigma_{1}, \Sigma_{2}, \Sigma_{3}\right)=\left(-m_{1}, m_{2}, m_{3}\right), \\
& \langle 6\rangle:\left(\Sigma_{1}, \Sigma_{2}, \Sigma_{3}\right)=\left(-m_{1}, m_{2},-m_{3}\right), \\
& \langle 7\rangle:\left(\Sigma_{1}, \Sigma_{2}, \Sigma_{3}\right)=\left(-m_{1},-m_{2}, m_{3}\right), \\
& \langle 8\rangle:\left(\Sigma_{1}, \Sigma_{2}, \Sigma_{3}\right)=\left(-m_{1},-m_{2},-m_{3}\right) .
\end{aligned}
$$

The tensions of elementary walls that connect vacua (3.34) are

$$
\begin{aligned}
T_{\langle 1 \leftarrow 2\rangle}=T_{\langle 3 \leftarrow 4\rangle}=T_{\langle 5 \leftarrow 6\rangle} & =T_{\langle 7 \leftarrow 8\rangle}=\vec{m} \cdot \vec{\alpha}_{3}, \\
T_{\langle 2 \leftarrow 3\rangle} & =T_{\langle 6 \leftarrow 7\rangle}=2 \vec{m} \cdot \vec{\alpha}_{2}, \\
T_{\langle 3 \leftarrow 5\rangle} & =T_{\langle 4 \leftarrow 6\rangle}=2 \vec{m} \cdot \vec{\alpha}_{1} .
\end{aligned}
$$

Therefore, the elementary walls are

$$
\begin{aligned}
\vec{g}_{\langle 1 \leftarrow 2\rangle}=\vec{g}_{\langle 3 \leftarrow 4\rangle}=\vec{g}_{\langle 5 \leftarrow 6\rangle} & =\vec{g}_{\langle 7 \leftarrow 8\rangle}=\vec{\alpha}_{3}, \\
\vec{g}_{\langle 2 \leftarrow 3\rangle} & =\vec{g}_{\langle 6 \leftarrow 7\rangle}=2 \vec{\alpha}_{2}, \\
\vec{g}_{\langle 3 \leftarrow 5\rangle} & =\vec{g}_{\langle 4 \leftarrow 6\rangle}=2 \vec{\alpha}_{1} .
\end{aligned}
$$

There are penetrable walls since $\vec{\alpha}_{1} \cdot \vec{\alpha}_{3}=0$. The diagram of the elementary walls of the nonlinear sigma model on $S p(3) / U(3)$ are depicted in Fig. 1(c). In this figure, a pair of penetrable elementary walls makes a parallelogram. A pair of facing sides of the parallelogram are the same simple roots, whereas a pair of adjacent sides of the parallelogram are orthogonal simple roots.

We make some observations of the walls. One can guess the existence of compressed walls from the wall diagram in Fig. 1(c). Since $\vec{g}_{\langle 1 \leftarrow 2\rangle} \cdot \vec{g}_{\langle 2 \leftarrow 3\rangle} \neq 0$, elementary wall $\langle 1 \leftarrow 2\rangle$ and elementary wall $\langle 2 \leftarrow 3\rangle$ are compressed to compressed wall $\langle 1 \leftarrow 3\rangle$, which is a compressed wall of level 1 . The moduli matrix of $\langle 1 \leftarrow 3\rangle$ is

$$
H_{0\langle 1 \leftarrow 3\rangle}=H_{0\langle 1\rangle} e^{\left[\left[E_{3}, E_{2}\right], E_{2}\right](r)} .
$$

One can also see that $\vec{g}_{\langle 2 \leftarrow 3\rangle} \cdot \vec{g}_{\langle 3 \leftarrow 5\rangle} \neq 0$. Therefore, elementary wall $\langle 2 \leftarrow 3\rangle$ and elementary wall $\langle 3 \leftarrow 5\rangle$ are compressed to compressed wall $\langle 2 \leftarrow 5\rangle$, which is a compressed wall of level 1 . The moduli matrix of compressed wall $\langle 2 \leftarrow 5\rangle$ is

$$
H_{0\langle 2 \leftarrow 5\rangle}=H_{0\langle 2\rangle} e^{\left[E_{2}, E_{1}\right](r)} .
$$

Let us consider the moduli matrix of double wall $\langle 1 \leftarrow 2 \leftarrow 3\rangle$,

$$
H_{0\langle 1 \leftarrow 2 \leftarrow 3\rangle}=H_{0\langle 1\rangle} e^{E_{3}\left(r_{1}\right)} e^{E_{2}\left(r_{2}\right)},
$$

and the moduli matrix of double wall $\langle 2 \leftarrow 3 \leftarrow 5\rangle$,

$$
H_{0\langle 2 \leftarrow 3 \leftarrow 5\rangle}=H_{0\langle 2\rangle} e^{E_{2}\left(r_{1}\right)} e^{E_{1}\left(r_{2}\right)} .
$$

Double wall $\langle 1 \leftarrow 2 \leftarrow 3\rangle$ in (3.39) is

$$
\begin{aligned}
H_{0\langle 1 \leftarrow 2 \leftarrow 3\rangle}= & H_{0\langle 1\rangle} e^{E_{3}\left(r_{1}\right)} e^{E_{2}\left(r_{2}\right)} \\
= & H_{0\langle 1\rangle} e^{E_{2}\left(r_{2}\right)} e^{E_{3}\left(r_{1}\right)} e^{\left[E_{2}, E_{3}\right]\left(r_{1}+r_{2}+i \pi\right)} \\
& \times e^{\left[E_{2},\left[E_{2}, E_{3}\right]\right]\left(r_{1}+2 r_{2}-\ln 2\right)} \\
\simeq & H_{0\langle 1\rangle} e^{E_{3}\left(r-2 r_{2}+\ln 2\right)} e^{\left[E_{2}, E_{3}\right]\left(r-r_{2}+\ln 2+i \pi\right)} \\
& \times e^{\left[E_{2},\left[E_{2}, E_{3}\right]\right](r)},
\end{aligned}
$$

where $r:=r_{1}+2 r_{2}-\ln 2$ and $\simeq$ means

$$
H_{0\langle 1\rangle} e^{E_{2}\left(r_{2}\right)}=\left(\begin{array}{ccc}
1 & 0 & 0 \\
0 & 1 & e^{r_{2}} \\
0 & 0 & 1
\end{array}\right) H_{0\langle 1\rangle} \simeq H_{0\langle 1\rangle} .
$$

As $r_{2} \rightarrow+\infty$ with finite $r$, the limit of $H_{0\langle 1 \leftarrow 2 \leftarrow 3\rangle}$ equals $H_{0\langle 1 \leftarrow 3\rangle}$. Double wall $\langle 1 \leftarrow 2 \leftarrow 3\rangle$ is plotted in Fig. 2 .

Double wall $\langle 2 \leftarrow 3 \leftarrow 5\rangle$ (3.40) is

$$
\begin{aligned}
H_{0\langle 2 \leftarrow 3 \leftarrow 5\rangle} & =H_{0\langle 2\rangle} e^{E_{2}\left(r_{1}\right)} e^{E_{1}\left(r_{2}\right)} \\
& =H_{0\langle 2\rangle} e^{E_{1}\left(r_{2}\right)} e^{E_{2}\left(r_{1}\right)} e^{\left[E_{2}, E_{1}\right]\left(r_{1}+r_{2}\right)} \\
& \simeq H_{0\langle 2\rangle} e^{E_{2}\left(r_{1}\right)} e^{\left[E_{1}, E_{2}\right](r)},
\end{aligned}
$$

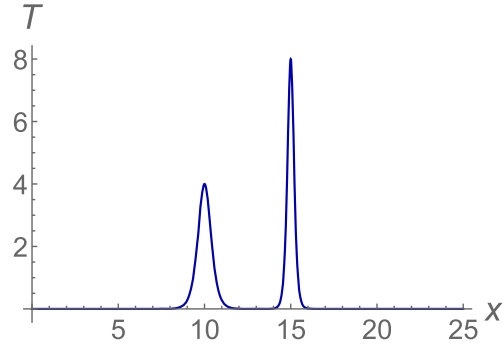

(a)

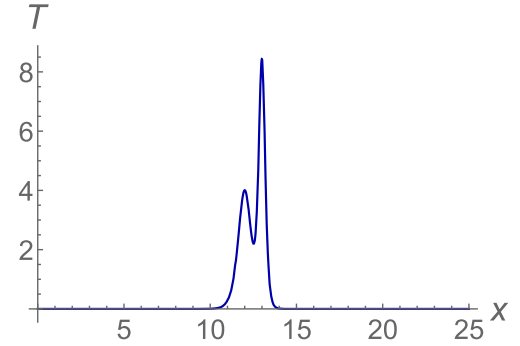

(b)

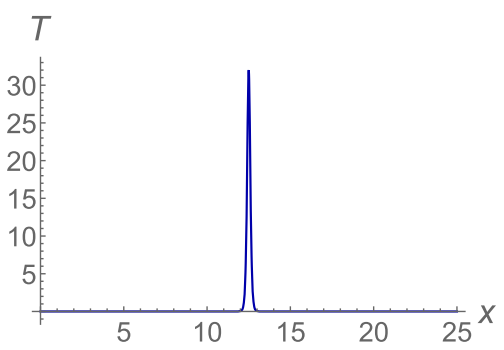

(c)

FIG. 2. Double wall $\langle 1 \leftarrow 2 \leftarrow 3\rangle$ in $S p(3) / U(3)$, which consists of two elementary walls $\langle 1 \leftarrow 2\rangle$ and $\langle 2 \leftarrow 3\rangle$. They are compressed to $\langle 1 \leftarrow 3\rangle \cdot m_{1}=8, m_{2}=4$, and $m_{3}=2$. (a) $r_{1}=60, r_{2}=20$; (b) $r_{1}=52, r_{2}=24$; and (c) $r_{1}=40, r_{2}=30$. 


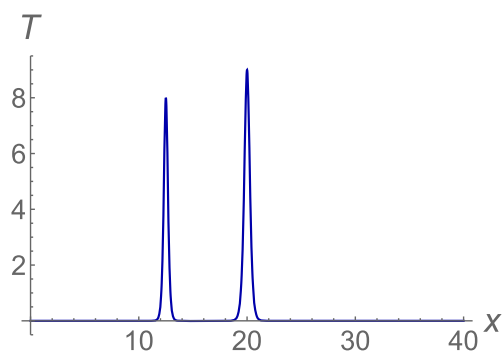

(a)

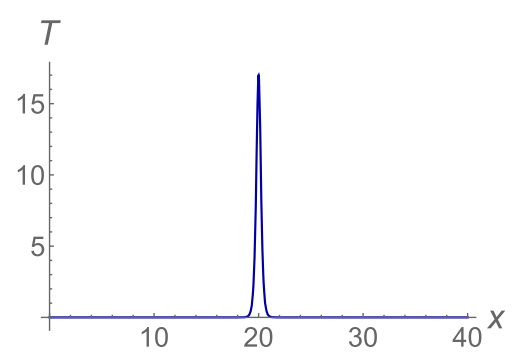

(b)

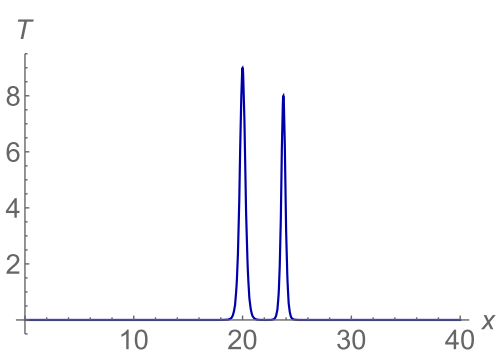

(c)

FIG. 3. Double wall $\langle 3 \leftarrow 5 \leftarrow 6\rangle$ in $S p(3) / U(3)$, which consists of two penetrable walls. $m_{1}=8, m_{2}=5$, and $m_{3}=2$. (a) $r_{1}=60$, $r_{2}=50$; (b) $r_{1}=60, r_{2}=80$; and (c) $r_{1}=60, r_{2}=95$.

where $r:=r_{1}+r_{2}+i \pi$ and $\simeq$ means

$$
H_{0\langle 2\rangle} e^{E_{1}\left(r_{2}\right)}=\left(\begin{array}{ccc}
1 & e^{r} & 0 \\
0 & 1 & 0 \\
0 & 0 & 1
\end{array}\right) H_{0\langle 2\rangle} \simeq H_{0\langle 2\rangle}
$$

As $r_{1} \rightarrow-\infty$ with finite $r$, the limit of $H_{0\langle 2 \leftarrow 3 \leftarrow 5\rangle}$ equals $H_{0\langle 2 \leftarrow 5\rangle}$. Double wall $\langle 2 \leftarrow 3 \leftarrow 5\rangle$ is compressed to compressed wall $\langle 2 \leftarrow 5\rangle$, which is a compressed wall of level 1.

Next, we discuss penetrable walls. Since $\vec{g}_{\langle 3 \leftarrow 5\rangle} \cdot \vec{g}_{\langle 5 \leftarrow 6\rangle}=0$, we can observe elementary wall $\langle 3 \leftarrow 5\rangle$ and elementary wall $\langle 5 \leftarrow 6\rangle$ pass through each other. Double wall $\langle 3 \leftarrow 5 \leftarrow 6\rangle$ is plotted in Fig. 3 .

The moduli matrix of $\langle 1 \leftarrow 5\rangle$, which is a compressed wall of level 2, is

$$
H_{0\langle 1 \leftarrow 5\rangle}=H_{0\langle 1\rangle} e^{\left.\left[\left[\left[E_{3}, E_{2}\right], E_{2}\right], E_{1}\right], E_{1}\right](r)} .
$$

The moduli matrix of triple wall $\langle 1 \leftarrow 2 \leftarrow 3 \leftarrow 5\rangle$ is

$$
H_{0\langle 1 \leftarrow 5\rangle}=H_{0\langle 1\rangle} e^{E_{3}\left(r_{1}\right)} e^{E_{2}\left(r_{2}\right)} e^{E_{1}\left(r_{3}\right)} .
$$

We shall consider higher $N$. Elementary walls can be identified with the simple roots of $U S p(2 N)$ with proper coefficients. All the compressed single walls and multiwalls can be constructed from the elementary wall configuration. The elementary wall configuration for $N=4$ is

$$
\begin{aligned}
\vec{g}_{\langle 5 \leftarrow 9\rangle} & =\vec{g}_{\langle 6 \leftarrow 10\rangle}=\vec{g}_{\langle 7 \leftarrow 11\rangle}=\vec{g}_{\langle 8 \leftarrow 12\rangle}=2 \vec{\alpha}_{1}, \\
\vec{g}_{\langle 3 \leftarrow 5\rangle} & =\vec{g}_{\langle 4 \leftarrow 6\rangle}=\vec{g}_{\langle 11 \leftarrow 13\rangle}=\vec{g}_{\langle 12 \leftarrow 14\rangle}=2 \vec{\alpha}_{2}, \\
\vec{g}_{\langle 2 \leftarrow 3\rangle} & =\vec{g}_{\langle 6 \leftarrow 7\rangle}=\vec{g}_{\langle 10 \leftarrow 11\rangle}=\vec{g}_{\langle 14 \leftarrow 15\rangle}=2 \vec{\alpha}_{3}, \\
\vec{g}_{\langle 1 \leftarrow 2\rangle} & =\vec{g}_{\langle 3 \leftarrow 4\rangle}=\vec{g}_{\langle 5 \leftarrow 6\rangle}=\vec{g}_{\langle 7 \leftarrow 8\rangle} \\
& =\vec{g}_{\langle 9 \leftarrow 10\rangle}=\vec{g}_{\langle 11 \leftarrow 12\rangle}=\vec{g}_{\langle 13 \leftarrow 14\rangle}=\vec{g}_{\langle 15 \leftarrow 16\rangle}=\vec{\alpha}_{4} .
\end{aligned}
$$

The diagram of the elementary walls is depicted in Fig. 1(d). We leave vacuum labels out of diagrams from Fig. 1(d) onward.

While the elementary wall diagrams are planar for $N \leq 4$, the diagrams are nonplanar for $N \geq 5$. The elementary wall configurations for $N=5$ and $N=6$ are as follows:

(i) $N=5$ :

$$
\begin{aligned}
\vec{g}_{\langle 9 \leftarrow 17\rangle} & =\vec{g}_{\langle 10 \leftarrow 18\rangle}=\vec{g}_{\langle 11 \leftarrow 19\rangle}=\vec{g}_{\langle 12 \leftarrow 20\rangle}=\vec{g}_{\langle 13 \leftarrow 21\rangle}=\vec{g}_{\langle 14 \leftarrow 22\rangle}=\vec{g}_{\langle 15 \leftarrow 23\rangle}=\vec{g}_{\langle 16 \leftarrow 24\rangle}=2 \vec{\alpha}_{1}, \\
\vec{g}_{\langle 5 \leftarrow 9\rangle} & =\vec{g}_{\langle 6 \leftarrow 10\rangle}=\vec{g}_{\langle 7 \leftarrow 11\rangle}=\vec{g}_{\langle 8 \leftarrow 12\rangle}=\vec{g}_{\langle 21 \leftarrow 25\rangle}=\vec{g}_{\langle 22 \leftarrow 26\rangle}=\vec{g}_{\langle 23 \leftarrow 27\rangle}=\vec{g}_{\langle 24 \leftarrow 28\rangle}=2 \vec{\alpha}_{2}, \\
\vec{g}_{\langle 3 \leftarrow 5\rangle} & =\vec{g}_{\langle 4 \leftarrow 6\rangle}=\vec{g}_{\langle 11 \leftarrow 13\rangle}=\vec{g}_{\langle 12 \leftarrow 14\rangle}=\vec{g}_{\langle 19 \leftarrow 21\rangle}=\vec{g}_{\langle 20 \leftarrow 22\rangle}=\vec{g}_{\langle 27 \leftarrow 29\rangle}=\vec{g}_{\langle 28 \leftarrow 30\rangle}=2 \vec{\alpha}_{3}, \\
\vec{g}_{\langle 2 \leftarrow 3\rangle} & =\vec{g}_{\langle 6 \leftarrow 7\rangle}=\vec{g}_{\langle 10 \leftarrow 11\rangle}=\vec{g}_{\langle 14 \leftarrow 15\rangle}=\vec{g}_{\langle 18 \leftarrow 19\rangle}=\vec{g}_{\langle 22 \leftarrow 23\rangle}=\vec{g}_{\langle 26 \leftarrow 27\rangle}=\vec{g}_{\langle 30 \leftarrow 31\rangle}=2 \vec{\alpha}_{4}, \\
\vec{g}_{\langle 1 \leftarrow 2\rangle} & =\vec{g}_{\langle 3 \leftarrow 4\rangle}=\vec{g}_{\langle 5 \leftarrow 6\rangle}=\vec{g}_{\langle 7 \leftarrow 8\rangle}=\vec{g}_{\langle 9 \leftarrow 10\rangle}=\vec{g}_{\langle 11 \leftarrow 12\rangle}=\vec{g}_{\langle 13 \leftarrow 14\rangle}=\vec{g}_{\langle 15 \leftarrow 16\rangle}=\vec{g}_{\langle 17 \leftarrow 18\rangle}=\vec{g}_{\langle 19 \leftarrow 20\rangle} \\
& =\vec{g}_{\langle 21 \leftarrow 22\rangle}=\vec{g}_{\langle 23 \leftarrow 24\rangle}=\vec{g}_{\langle 25 \leftarrow 26\rangle}=\vec{g}_{\langle 27 \leftarrow 28\rangle}=\vec{g}_{\langle 29 \leftarrow 30\rangle}=\vec{g}_{\langle 31 \leftarrow 32\rangle}=\vec{\alpha}_{5} .
\end{aligned}
$$


(ii) $N=6$ :

$$
\begin{aligned}
\vec{g}_{\langle 17 \leftarrow 33\rangle} & =\vec{g}_{\langle 18 \leftarrow 34\rangle}=\vec{g}_{\langle 19 \leftarrow 35\rangle}=\vec{g}_{\langle 20 \leftarrow 36\rangle}=\vec{g}_{\langle 21 \leftarrow 37\rangle}=\vec{g}_{\langle 22 \leftarrow 38\rangle}=\vec{g}_{\langle 23 \leftarrow 39\rangle}=\vec{g}_{\langle 24 \leftarrow 40\rangle}=\vec{g}_{\langle 25 \leftarrow 41\rangle}=\vec{g}_{\langle 26 \leftarrow 42\rangle} \\
& =\vec{g}_{\langle 27 \leftarrow 43\rangle}=\vec{g}_{\langle 28 \leftarrow 44\rangle}=\vec{g}_{\langle 29 \leftarrow 45\rangle}=\vec{g}_{\langle 30 \leftarrow 46\rangle}=\vec{g}_{\langle 31 \leftarrow 47\rangle}=\vec{g}_{\langle 32 \leftarrow 48\rangle}=2 \vec{\alpha}_{1}, \\
\vec{g}_{\langle 9 \leftarrow 17\rangle} & =\vec{g}_{\langle 10 \leftarrow 18\rangle}=\vec{g}_{\langle 11 \leftarrow 19\rangle}=\vec{g}_{\langle 12 \leftarrow 20\rangle}=\vec{g}_{\langle 13 \leftarrow 21\rangle}=\vec{g}_{\langle 14 \leftarrow 22\rangle}=\vec{g}_{\langle 15 \leftarrow 23\rangle}=\vec{g}_{\langle 16 \leftarrow 24\rangle} \\
& =\vec{g}_{\langle 41 \leftarrow 49\rangle}=\vec{g}_{\langle 42 \leftarrow 50\rangle}=\vec{g}_{\langle 43 \leftarrow 51\rangle}=\vec{g}_{\langle 44 \leftarrow 52\rangle}=\vec{g}_{\langle 45 \leftarrow 53\rangle}=\vec{g}_{\langle 46 \leftarrow 54\rangle}=\vec{g}_{\langle 47 \leftarrow 55\rangle}=\vec{g}_{\langle 48 \leftarrow 56\rangle}=2 \vec{\alpha}_{2}, \\
\vec{g}_{\langle 5 \leftarrow 9\rangle} & =\vec{g}_{\langle 6 \leftarrow 10\rangle}=\vec{g}_{\langle 7 \leftarrow 11\rangle}=\vec{g}_{\langle 8 \leftarrow 12\rangle}=\vec{g}_{\langle 21 \leftarrow 25\rangle}=\vec{g}_{\langle 22 \leftarrow 26\rangle}=\vec{g}_{\langle 23 \leftarrow 27\rangle}=\vec{g}_{\langle 24 \leftarrow 28\rangle} \\
& =\vec{g}_{\langle 37 \leftarrow 41\rangle}=\vec{g}_{\langle 38 \leftarrow 42\rangle}=\vec{g}_{\langle 39 \leftarrow 43\rangle}=\vec{g}_{\langle 40 \leftarrow 44\rangle}=\vec{g}_{\langle 53 \leftarrow 57\rangle}=\vec{g}_{\langle 54 \leftarrow 58\rangle}=\vec{g}_{\langle 55 \leftarrow 59\rangle}=\vec{g}_{\langle 56 \leftarrow 60\rangle}=2 \vec{\alpha}_{3}, \\
\vec{g}_{\langle 3 \leftarrow 5\rangle} & =\vec{g}_{\langle 4 \leftarrow 6\rangle}=\vec{g}_{\langle 11 \leftarrow 13\rangle}=\vec{g}_{\langle 12 \leftarrow 14\rangle}=\vec{g}_{\langle 19 \leftarrow 21\rangle}=\vec{g}_{\langle 20 \leftarrow 22\rangle}=\vec{g}_{\langle 27 \leftarrow 29\rangle}=\vec{g}_{\langle 28 \leftarrow 30\rangle}=\vec{g}_{\langle 35 \leftarrow 37\rangle}=\vec{g}_{\langle 36 \leftarrow 38\rangle} \\
& =\vec{g}_{\langle 43 \leftarrow 45\rangle}=\vec{g}_{\langle 44 \leftarrow 46\rangle}=\vec{g}_{\langle 51 \leftarrow 53\rangle}=\vec{g}_{\langle 52 \leftarrow 54\rangle}=\vec{g}_{\langle 59 \leftarrow 61\rangle}=\vec{g}_{\langle 60 \leftarrow 62\rangle}=2 \vec{\alpha}_{4}, \\
\vec{g}_{\langle 2 \leftarrow 3\rangle} & =\vec{g}_{\langle 6 \leftarrow 7\rangle}=\vec{g}_{\langle 10 \leftarrow 11\rangle}=\vec{g}_{\langle 14 \leftarrow 15\rangle}=\vec{g}_{\langle 18 \leftarrow 19\rangle}=\vec{g}_{\langle 22 \leftarrow 23\rangle}=\vec{g}_{\langle 26 \leftarrow 27\rangle}=\vec{g}_{\langle 30 \leftarrow 31\rangle} \\
& =\vec{g}_{\langle 34 \leftarrow 35\rangle}=\vec{g}_{\langle 38 \leftarrow 39\rangle}=\vec{g}_{\langle 42 \leftarrow 43\rangle}=\vec{g}_{\langle 46 \leftarrow 47\rangle}=\vec{g}_{\langle 50 \leftarrow 51\rangle}=\vec{g}_{\langle 54 \leftarrow 55\rangle}=\vec{g}_{\langle 58 \leftarrow 59\rangle}=\vec{g}_{\langle 62 \leftarrow 63\rangle}=2 \vec{\alpha}_{5}, \\
& =\vec{g}_{\langle 3 \leftarrow 4\rangle}=\vec{g}_{\langle 5 \leftarrow 6\rangle}=\vec{g}_{\langle 7 \leftarrow 8\rangle}=\vec{g}_{\langle 9 \leftarrow 10\rangle}=\vec{g}_{\langle 11 \leftarrow 12\rangle}=\vec{g}_{\langle 13 \leftarrow 14\rangle}=\vec{g}_{\langle 15 \leftarrow 16\rangle}=\vec{g}_{\langle 17 \leftarrow 18\rangle}=\vec{g}_{\langle 19 \leftarrow 20\rangle}=\vec{g}_{\langle 21 \leftarrow 22\rangle} \\
& =\vec{g}_{\langle 23 \leftarrow 24\rangle}=\vec{g}_{\langle 25 \leftarrow 26\rangle}=\vec{g}_{\langle 27 \leftarrow 28\rangle}=\vec{g}_{\langle 29 \leftarrow 30\rangle}=\vec{g}_{\langle 31 \leftarrow 32\rangle}=\vec{g}_{\langle 33 \leftarrow 34\rangle}=\vec{g}_{\langle 35 \leftarrow 36\rangle}=\vec{g}_{\langle 37 \leftarrow 38\rangle}=\vec{g}_{\langle 39 \leftarrow 40\rangle} \\
& =\vec{g}_{\langle 41 \leftarrow 42\rangle}=\vec{g}_{\langle 43 \leftarrow 44\rangle}=\vec{g}_{\langle 45 \leftarrow 46\rangle}=\vec{g}_{\langle 47 \leftarrow 48\rangle}=\vec{g}_{\langle 49 \leftarrow 50\rangle}=\vec{g}_{\langle 51 \leftarrow 52\rangle}=\vec{g}_{\langle 53 \leftarrow 54\rangle}=\vec{g}_{\langle 55 \leftarrow 56\rangle} \\
& =\vec{g}_{\langle 57 \leftarrow 58\rangle}=\vec{g}_{\langle 59 \leftarrow 60\rangle}=\vec{g}_{\langle 61 \leftarrow 62\rangle}=\vec{g}_{\langle 63 \leftarrow 64\rangle}=\vec{\alpha}_{6} .
\end{aligned}
$$

The diagrams of the elementary walls of the $N=5$ and $N=6$ cases are depicted in Figs. 4 and 5.

\section{VACUA CONNECTED TO THE MAXIMUM NUMBER OF ELEMENTARY WALLS}

We study the vacua that are connected to the maximum number of elementary walls. We denote the vacua $\langle A\rangle$ and

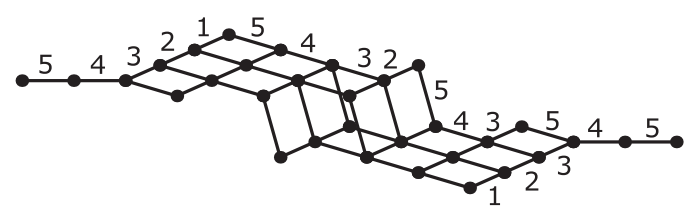

FIG. 4. Elementary walls of the nonlinear sigma model on $S p(5) / U(5)$. The left-hand side is the limit as $x \rightarrow+\infty$, and the right-hand side is the limit as $x \rightarrow-\infty$.

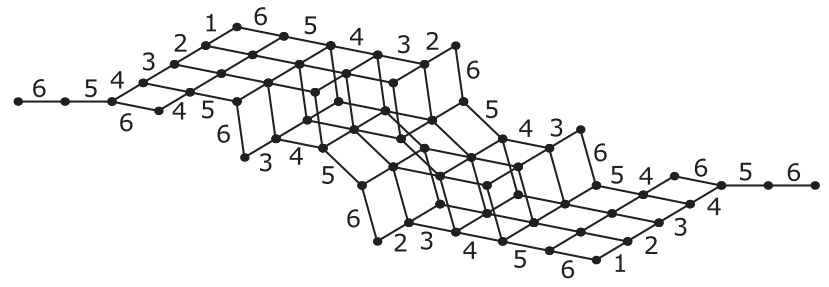

FIG. 5. Elementary walls on $S p(6) / U(6)$. The left-hand side is the limit as $x \rightarrow+\infty$, and the right-hand side is the limit as $x \rightarrow-\infty$. $\langle B\rangle$. Let $\langle A\rangle$ be the vacuum near $\langle 1\rangle$ and $\langle B\rangle$ be the vacuum near $\left\langle 2^{N}\right\rangle$. From Figs. 1, 4, and 5, we make the following observations in which $\vec{m}$ denotes simple root $\vec{\alpha}_{m}$ :

(i) $N=1$ :

$$
\langle 1\rangle \leftarrow \overrightarrow{1} \leftarrow\langle 2\rangle .
$$

(ii) $N=2$ :

$$
\overrightarrow{2} \leftarrow\langle A\rangle \leftarrow \overrightarrow{1} \leftarrow\langle B\rangle \leftarrow \overrightarrow{2} .
$$

(iii) $N=3$ :

$$
\begin{aligned}
\overrightarrow{3} \leftarrow \cdots & \leftarrow \overrightarrow{2} \leftarrow\langle A\rangle \leftarrow\{\overrightarrow{1}, \overrightarrow{3}\} \leftarrow \cdots \\
& \quad \cdots \leftarrow\{\overrightarrow{1}, \overrightarrow{3}\} \leftarrow\langle B\rangle \leftarrow \overrightarrow{2} \leftarrow \cdots \leftarrow \overrightarrow{3} .
\end{aligned}
$$

(iv) $N=4$ :

$$
\begin{aligned}
\overrightarrow{4} \leftarrow \cdots & \leftarrow\{\overrightarrow{2}, \overrightarrow{4}\} \leftarrow\langle A\rangle \leftarrow\{\overrightarrow{1}, \overrightarrow{3}\} \leftarrow \cdots \\
& \cdots \leftarrow\{\overrightarrow{1}, \overrightarrow{3}\} \leftarrow\langle B\rangle \leftarrow\{\overrightarrow{2}, \overrightarrow{4}\} \leftarrow \cdots \leftarrow \overrightarrow{4} .
\end{aligned}
$$

(v) $N=5$ :

$$
\begin{aligned}
\overrightarrow{5} \leftarrow \cdots & \leftarrow\{\overrightarrow{2}, \overrightarrow{4}\} \leftarrow\langle A\rangle \leftarrow\{\overrightarrow{1}, \overrightarrow{3}, \overrightarrow{5}\} \leftarrow \cdots \\
& \cdots \leftarrow\{\overrightarrow{1}, \overrightarrow{3}, \overrightarrow{5}\} \leftarrow\langle B\rangle \leftarrow\{\overrightarrow{2}, \overrightarrow{4}\} \leftarrow \cdots \leftarrow \overrightarrow{5} .
\end{aligned}
$$


(vi) $N=6$ :

$$
\begin{aligned}
\overrightarrow{6} \leftarrow \cdots & \leftarrow\{\overrightarrow{2}, \overrightarrow{4}, \overrightarrow{6}\} \leftarrow\langle A\rangle \leftarrow\{\overrightarrow{1}, \overrightarrow{3}, \overrightarrow{5}\} \leftarrow \cdots \\
& \cdots \leftarrow\{\overrightarrow{1}, \overrightarrow{3}, \overrightarrow{5}\} \leftarrow\langle B\rangle \leftarrow\{\overrightarrow{2}, \overrightarrow{4}, \overrightarrow{6}\} \leftarrow \cdots \leftarrow \overrightarrow{6} .
\end{aligned}
$$

From Figs. 1, 4, and 5 and Eqs. (3.36), (3.47), (3.48), and (3.49), $\langle A\rangle$ and $\langle B\rangle$ are identified as follows:

(i) $N=3$ :

$$
\langle A\rangle=\langle 3\rangle, \quad\langle B\rangle=\langle 6\rangle .
$$

(ii) $N=4$ :

$$
\langle A\rangle=\langle 6\rangle, \quad\langle B\rangle=\langle 11\rangle .
$$

(iii) $N=5$ :

$$
\langle A\rangle=\langle 11\rangle, \quad\langle B\rangle=\langle 22\rangle .
$$

(iv) $N=6$ :

$$
\langle A\rangle=\langle 22\rangle, \quad\langle B\rangle=\langle 43\rangle .
$$

The vacuum labels are not unique since we can change them as we please. Therefore, let us label the vacua that are connected to the maximum number of elementary walls as $\langle A\rangle$ and $\langle B\rangle$.

The vacuum structures that are connected to the maximum number of elementary walls are as follows:

(i) $N=4 m-3(m \geq 2)$ :

$$
\begin{aligned}
\vec{N} \leftarrow \cdots & \\
& \cdots \leftarrow\{\underbrace{\overrightarrow{2}, \overrightarrow{4}, \ldots, \overrightarrow{4 m-6}, \overrightarrow{4 m-4}}_{2 m-2}\} \leftarrow\langle A\rangle \leftarrow \\
& \leftarrow\{\underbrace{\overrightarrow{1}, \overrightarrow{3}, \ldots, \overrightarrow{4 m-5}, \overrightarrow{4 m-3}}_{2 m-1}\} \leftarrow \cdots \\
& \cdots \leftarrow\{\underbrace{\overrightarrow{1}, \overrightarrow{3}, \ldots, \overrightarrow{4 m-5}, \overrightarrow{4 m-3}}_{2 m-1}\} \leftarrow\langle B\rangle \leftarrow \\
& \leftarrow\{\underbrace{\overrightarrow{2}, \overrightarrow{4}, \ldots, \overrightarrow{4 m-6}, \overrightarrow{4 m-4}}_{2 m-2}\} \leftarrow \cdots \\
& \cdots \leftarrow \leftarrow \vec{N} .
\end{aligned}
$$

(ii) $N=4 m-2(m \geq 2)$ :

$$
\begin{aligned}
\vec{N} \leftarrow \cdots & \\
\cdots & \leftarrow\{\underbrace{\overrightarrow{2}, \overrightarrow{4}, \ldots, \overrightarrow{4 m-4}, \overrightarrow{4 m-2}}_{2 m-1}\} \leftarrow\langle A\rangle \leftarrow \\
& \leftarrow\{\underbrace{\overrightarrow{1}, \overrightarrow{3}, \ldots, \overrightarrow{4 m-5}, \overrightarrow{4 m-3}}_{2 m-1}\} \leftarrow \cdots \\
\cdots & \leftarrow\{\underbrace{\overrightarrow{1}, \overrightarrow{3}, \ldots, \overrightarrow{4 m-5}, \overrightarrow{4 m-3}}_{2 m-1}\} \leftarrow\langle B\rangle \leftarrow \\
& \leftarrow\{\underbrace{\overrightarrow{2}, \overrightarrow{4}, \ldots, \overrightarrow{4 m-4}, \overrightarrow{4 m-2}}_{2 m-1}\} \leftarrow \cdots \\
\cdots & \leftarrow \vec{N} .
\end{aligned}
$$

(iii) $N=4 m-1(m \geq 2)$ :

$\vec{N} \leftarrow \cdots$

$$
\begin{aligned}
& \cdots \leftarrow\{\underbrace{\overrightarrow{2}, \overrightarrow{4}, \ldots, \overrightarrow{4 m-4}, \overrightarrow{4 m-2}}_{2 m-1}\} \leftarrow\langle A\rangle \leftarrow \\
\leftarrow & \{\underbrace{\overrightarrow{1}, \overrightarrow{3}, \ldots, \overrightarrow{4 m-3}, \overrightarrow{4 m-1}}_{2 m}\} \leftarrow \cdots \\
& \cdots \leftarrow\{\underbrace{\overrightarrow{1}, \overrightarrow{3}, \ldots, \overrightarrow{4 m-3}, \overrightarrow{4 m-1}}_{2 m}\} \leftarrow\langle B\rangle \leftarrow \\
\leftarrow & \{\underbrace{\overrightarrow{2}, \overrightarrow{4}, \ldots, \overrightarrow{4 m-2}, \overrightarrow{4 m}}_{2 m}\} \leftarrow \cdots \\
& \quad \cdots \leftarrow \vec{N} .
\end{aligned}
$$

(iv) $N=4 m(m \geq 2)$ :

$$
\begin{aligned}
\vec{N} \leftarrow & \cdots \\
& \cdots \leftarrow\{\underbrace{\overrightarrow{2}, \overrightarrow{4}, \ldots, \overrightarrow{4 m-2}, \overrightarrow{4 m}}_{2 m}\} \leftarrow\langle A\rangle \leftarrow \\
\leftarrow & \{\underbrace{\overrightarrow{1}, \overrightarrow{3}, \ldots, \overrightarrow{4 m-3}, \overrightarrow{4 m-1}}_{2 m}\} \leftarrow \cdots \\
& \cdots \leftarrow\{\underbrace{\overrightarrow{1}, \overrightarrow{3}, \ldots, \overrightarrow{4 m-3}, \overrightarrow{4 m-1}}_{2 m}\} \leftarrow\langle B\rangle \leftarrow \\
\leftarrow & \{\underbrace{\overrightarrow{2}, \overrightarrow{4}, \ldots, \overrightarrow{4 m-2}, \overrightarrow{4 m}}_{2 m}\} \leftarrow \cdots \\
& \cdots \leftarrow \vec{N} .
\end{aligned}
$$

Equations (4.11), (4.12), (4.13), and (4.14) are proven in the Appendix. 


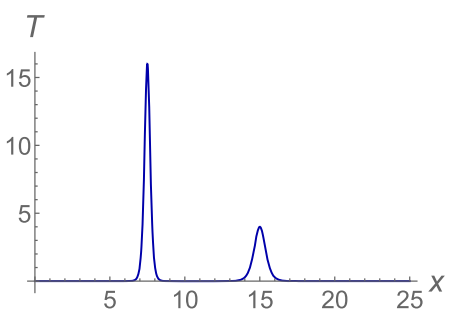

(a)

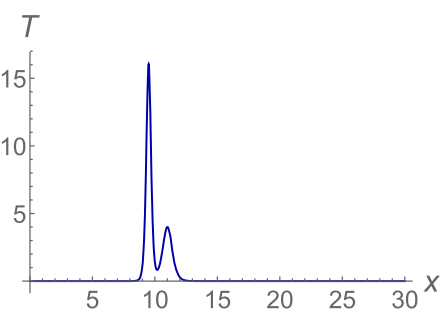

(b)

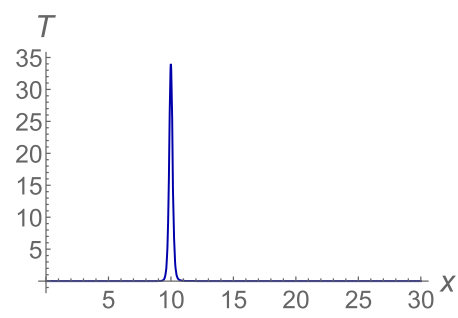

(c)

FIG. 6. Double wall $\langle 7 \leftarrow 11 \leftarrow 19\rangle$ in $S p(5) / U(5)$. Elementary walls $\langle 7 \leftarrow 11\rangle$ and $\langle 11 \leftarrow 19\rangle$ are compressed to $\langle 7 \leftarrow 19\rangle$ as $r_{1} \rightarrow-\infty$ with $r_{1}+r_{2}=r$ (finite). $m_{1}=12, m_{2}=8, m_{3}=6, m_{4}=4$, and $m_{5}=2$. (a) $r_{1}=30, r_{2}=30$; (b) $r_{1}=22, r_{2}=38$; and (c) $r_{1}=19, r_{2}=41$.

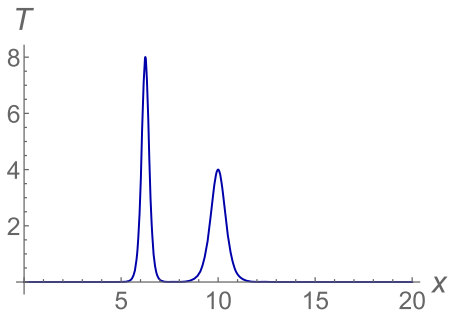

(a)

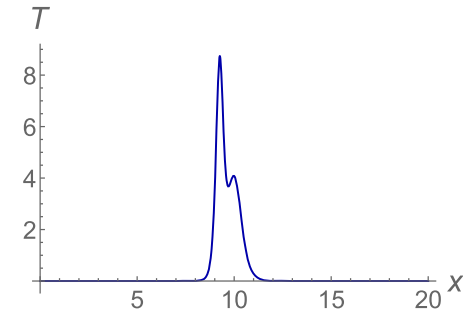

(b)

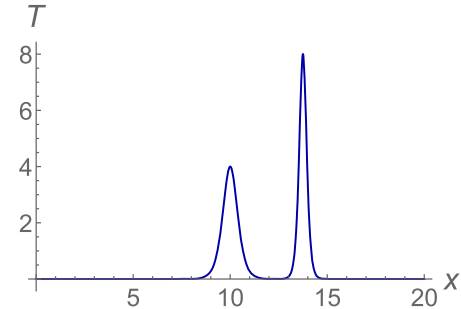

(c)

FIG. 7. Double wall $\langle 7 \leftarrow 11 \leftarrow 12\rangle$ in $S p(5) / U(5)$. Elementary walls $\langle 7 \leftarrow 11\rangle$ and $\langle 11 \leftarrow 12\rangle$ pass through each other. $m_{1}=12$, $m_{2}=8, m_{3}=6, m_{4}=4$, and $m_{5}=2$. (a) $r_{1}=20, r_{2}=25$; (b) $r_{1}=20, r_{2}=37$; and (c) $r_{1}=20, r_{2}=55$.

\section{WALLS OF NONLINEAR SIGMA MODEL ON $S p(5) / U(5)$}

We have studied the vacuum structures that are connected to the maximum number of elementary walls for general $N$. The elementary walls can be compressed or can pass through each other. We discuss some features of elementary walls of the nonlinear sigma model on $S p(5) / U(5)$, which is the simplest nontrivial case. From (3.48) and (4.5), $\langle 11\rangle$ is one of the vacua that are connected to the maximum number of elementary walls. The structure near $\langle 11\rangle$ is

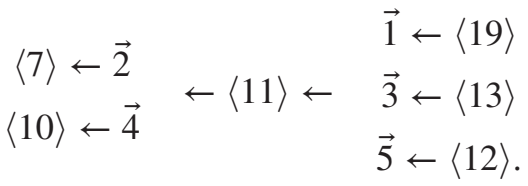

In (5.1), $\vec{\alpha}_{2} \cdot \vec{\alpha}_{1} \neq 0$. Therefore, elementary wall $\langle 7 \leftarrow 11\rangle$ and elementary wall $\langle 11 \leftarrow 19\rangle$ are compressed to a single wall. Vacuum $\langle 7\rangle$ is labeled by $\left(\Sigma_{1}, \Sigma_{2}, \Sigma_{3}, \Sigma_{4}, \Sigma_{5}\right)=$ $\left(m_{1}, m_{2},-m_{3},-m_{4}, m_{5}\right)$. The moduli matrix for double wall $\langle 7 \leftarrow 11 \leftarrow 19\rangle$ is

$$
\begin{aligned}
H_{0\langle 7 \leftarrow 11 \leftarrow 19\rangle} & =H_{0\langle 7\rangle} e^{E_{2}\left(r_{1}\right)} e^{E_{1}\left(r_{2}\right)} \\
& =H_{0\langle 7\rangle} e^{E_{1}\left(r_{2}\right)} e^{E_{2}\left(r_{1}\right)-\left[E_{1}, E_{2}\right]\left(r_{1}+r_{2}\right)} \\
& \simeq H_{0\langle 7\rangle} e^{E_{2}\left(r_{1}\right)-\left[E_{1}, E_{2}\right]\left(r_{1}+r_{2}\right)},
\end{aligned}
$$

where $\simeq$ means

$$
H_{0\langle>\rangle} e^{E_{1}\left(r_{2}\right)}=\left(I_{5}+e^{r} e_{1,2}\right) H_{0\langle 7\rangle} \simeq H_{0\langle 7\rangle} .
$$

As $r_{1} \rightarrow-\infty$ with $r_{1}+r_{2}=r$ (finite), $H_{0\langle 7 \leftarrow 11 \leftarrow 19\rangle} \rightarrow$ $H_{0\langle 7 \leftarrow 19\rangle}$. Double wall $\langle 7 \leftarrow 11 \leftarrow 19\rangle$ is compressed to compressed wall $\langle 7 \leftarrow 19\rangle$, which is a compressed wall of level 1. This is depicted in Fig. 6.

In (5.1), $\vec{\alpha}_{2} \cdot \vec{\alpha}_{5}=0$. Therefore, elementary wall $\langle 7 \leftarrow 11\rangle$ and elementary wall $\langle 11 \leftarrow 12\rangle$ are penetrable. The moduli matrix of double wall $\langle 7 \leftarrow 11 \leftarrow 12\rangle$, which consists of two penetrable elementary walls $\langle 7 \leftarrow 11\rangle$ and $\langle 11 \leftarrow 12\rangle$, is

$$
\begin{aligned}
& H_{0\langle 7 \leftarrow 11 \leftarrow 12\rangle} \\
& =H_{0\langle 7 \leftarrow 11\rangle} e^{E_{5}\left(r_{2}\right)}=H_{0\langle 7\rangle} e^{E_{2}\left(r_{1}\right)} e^{E_{5}\left(r_{2}\right)}=H_{0\langle 7\rangle} e^{E_{5}\left(r_{2}\right)} e^{E_{2}\left(r_{1}\right)} \\
& =H_{0\langle 7 \leftarrow 8\rangle} e^{E_{2}\left(r_{1}\right)} .
\end{aligned}
$$

This is depicted in Fig. 7.

\section{CONCLUSION}

We have studied the vacua and the walls of massdeformed Kähler nonlinear sigma models on $S p(N) /$ $U(N)$ by using the moduli matrix formalism. For $N=1$ and $N=2$, the nonlinear sigma models on $\operatorname{Sp}(N) / U(N)$ are Abelian theories, in which single walls are compressed to compressed walls while penetrable walls are not allowed. 
On the other hand, for $N \geq 3$, the nonlinear sigma models on $\operatorname{Sp}(N) / U(N)$ are non-Abelian theories, so there exist penetrable walls, which lead to a unique vacuum configuration for each $N$. We have proven the vacuum structures that are connected to the maximum number of elementary walls by induction.

\section{ACKNOWLEDGMENTS}

We thank B. -H. Lee for early participation. We would like to thank S. Krivonos, A. Sutulin, and N. Tyurin for helpful comments. A. G. would also like to thank the Asia Pacific Center for Theoretical Physics APCTP, Pohang, for warm hospitality. C. P. was supported by Basic Science Research Program through the National Research Foundation of Korea funded by the Ministry of Education (Grant No. NRF-2016R1D1A1B03932371). S. S. is supported by Basic Science Research Program through the National Research Foundation of Korea (Grant No. NRF-2017R1D1A1B03034222).

\section{APPENDIX: VACUUM STRUCTURES}

In this Appendix, we prove (4.11), (4.12), (4.13), and (4.14). The vacuum structures that are connected to the maximum number of elementary walls in the nonlinear sigma models on $S O(2 N) / U(N)$ are studied by decomposing the diagrams into two-dimensional diagrams in Ref. [11]. We use the same method in the nonlinear sigma models on $\operatorname{Sp}(N) / U(N)$. The rule for the decomposition is

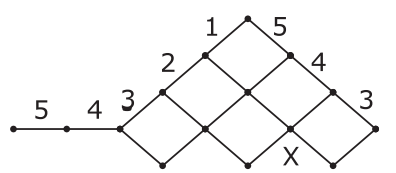

(a)

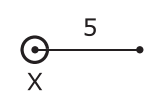

(b)
FIG. 8. $N=5$ case. The vacuum structure near $\langle 1\rangle(\langle 32\rangle)$ decomposes into two diagrams.

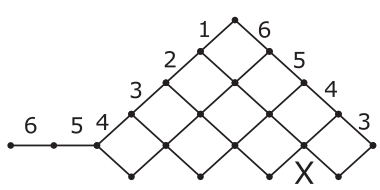

(a)

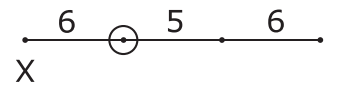

(b)
FIG. 9. $N=6$ case. The vacuum structure near $\langle 1\rangle(\langle 64\rangle)$ decomposes into two diagrams.

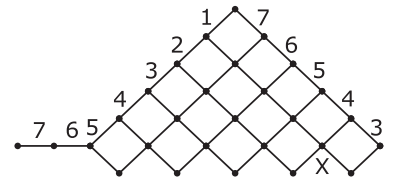

(a)

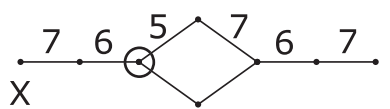

(b)
FIG. 10. $N=7$ case. The vacuum structure near $\langle 1\rangle(\langle 128\rangle)$ decomposes into two diagrams. that the simple roots that have already appeared in the previous diagrams should not be repeated.

The vacuum structure of the $N=5$ case is depicted in Fig. 4. The vacuum structure near $\langle 1\rangle(\langle 32\rangle)$ decomposes into two diagrams, as is shown in Fig. 8. The circle indicates $\langle A\rangle(\langle B\rangle)$. The letter $\mathrm{X}$ indicates the vacuum that is connected to the both diagrams. The left-hand side (the right-hand side) of the each diagram is the limit as $x \rightarrow$ $+\infty(x \rightarrow-\infty)$ for the vacuum structure near $\langle 1\rangle$, whereas the left-hand side (the right-hand side) of each diagram is the limit as $x \rightarrow-\infty(x \rightarrow+\infty)$ for the vacuum structure near $\langle 32\rangle$.

Figure 5, which describes the vacuum structure of the $N=6$, case decomposes into two diagrams as is shown in Fig. 9. In the same manner, the vacuum structures of the $N=7$ and $N=8$ cases are presented in Figs. 10 and 11. The vacuum structures repeat the four diagrams in Fig. 1.

All the vacuum structures can be decomposed into twodimensional diagrams in Fig. 12, in which only the first two diagrams are shown and then fall into four categories. The vacuum that is connected to the maximum number of elementary walls is circled in each diagram in Fig. 13.

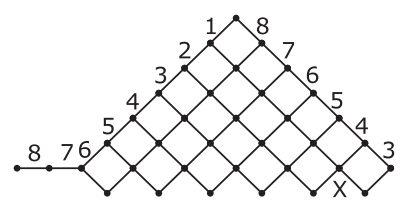

(a)

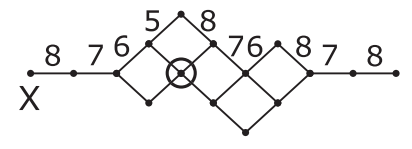

(b)
FIG. 11. $N=8$ case. The vacuum structure near $\langle 1\rangle(\langle 256\rangle)$ decomposes into two diagrams.

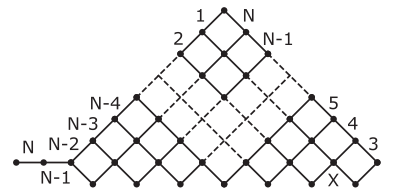

(a)

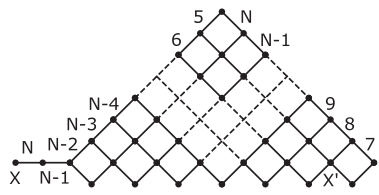

(b)
FIG. 12. First two diagrams of the vacuum structure near $\langle 1\rangle$ and $\left\langle 2^{N}\right\rangle$.

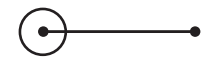

(a)

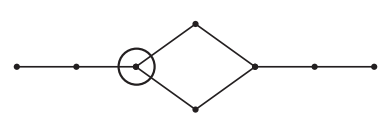

(c)

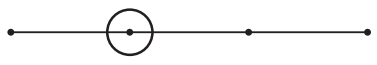

(b)

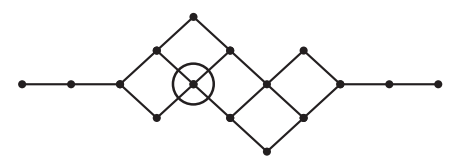

(d)
FIG. 13. Four types of vacuum structures. The circle indicates the vacuum that is connected to the maximum number of simple roots. 


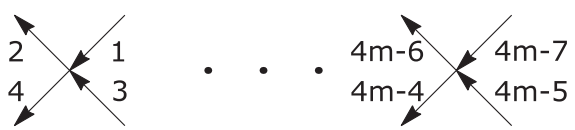

FIG. 14. Common part near $\langle A\rangle$.

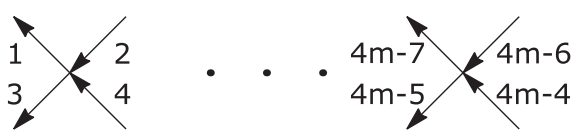

FIG. 15. Common part near $\langle B\rangle$.

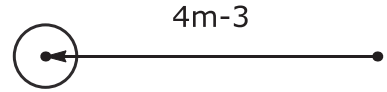

(a)

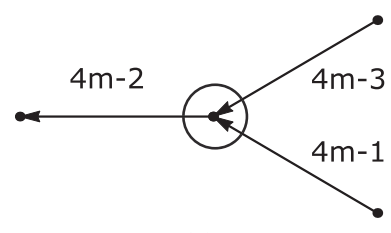

(c)

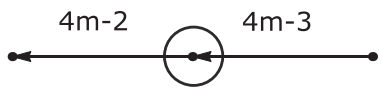

(b)

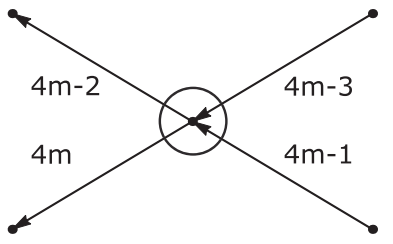

(d)

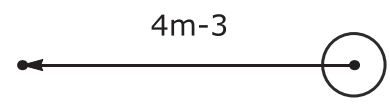

(a)

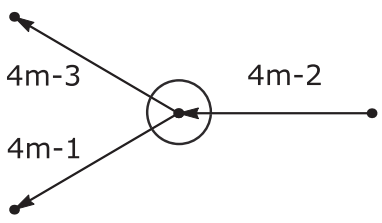

(c)

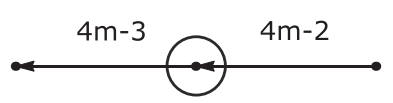

(b)

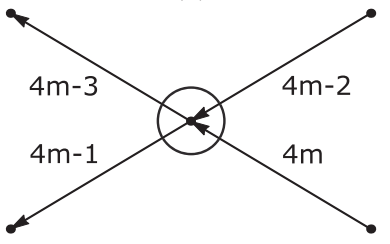

(d)
FIG. 17. Remaining part of the vacuum structure near $\langle B\rangle$. The location of $\langle B\rangle$ is circled.

The vacuum structures that are connected to the maximum number of elementary walls can be obtained from the repeated diagrams. $\langle A\rangle$ denotes the vacuum near $\langle 1\rangle$, and $\langle B\rangle$ denotes the vacuum near $\left\langle 2^{N}\right\rangle$. The common parts of each vacuum structure near $\langle A\rangle$ and $\langle B\rangle$ are shown in Figs. 14 and 15. The rest of the vacuum structures are obtained from Fig. 13. The remaining parts of each vacuum structure near $\langle A\rangle$ and $\langle B\rangle$ are shown in Figs. 16 and 17 for $N=4 m-3, N=4 m-2, N=4 m-1$, and $N=4 m$.

The vacuum structure of $\langle A\rangle$ is derived from Figs. 14 and 16 as follows:

location of $\langle A\rangle$ is circled.

(i) $N=4 m-3,(m \geq 2)$ :

$$
\{\underbrace{\overrightarrow{2}, \overrightarrow{4}, \ldots, \overrightarrow{4 m-6}, \overrightarrow{4 m-4}}_{2 m-2}\} \leftarrow\langle A\rangle \leftarrow\{\underbrace{\overrightarrow{1}, \overrightarrow{3}, \ldots, \overrightarrow{4 m-5}, \overrightarrow{4 m-3}}_{2 m-1}\} .
$$

(ii) $N=4 m-2,(m \geq 2)$ :

$$
\{\underbrace{\overrightarrow{2}, \overrightarrow{4}, \ldots, \overrightarrow{4 m-4}, \overrightarrow{4 m-2}}_{2 m-1}\} \leftarrow\langle A\rangle \leftarrow\{\underbrace{\overrightarrow{1}, \overrightarrow{3}, \ldots, \overrightarrow{4 m-5}, \overrightarrow{4 m-3}}_{2 m-1}\} .
$$

(iii) $N=4 m-1,(m \geq 2)$ :

$$
\{\underbrace{\overrightarrow{2}, \overrightarrow{4}, \ldots, \overrightarrow{4 m-4}, \overrightarrow{4 m-2}}_{2 m-1}\} \leftarrow\langle A\rangle \leftarrow\{\underbrace{\overrightarrow{1}, \overrightarrow{3}, \ldots, \overrightarrow{4 m-3}, \overrightarrow{4 m-1}}_{2 m}\} .
$$

(iv) $N=4 m,(m \geq 2)$ :

$$
\{\underbrace{\overrightarrow{2}, \overrightarrow{4}, \ldots, \overrightarrow{4 m-2}, \overrightarrow{4 m}}_{2 m}\} \leftarrow\langle A\rangle \leftarrow\{\underbrace{\overrightarrow{1}, \overrightarrow{3}, \ldots, \overrightarrow{4 m-3}, \overrightarrow{4 m-1}}_{2 m}\} .
$$

Each case with $m=1$ is shown in Fig. 1. Each case with $m=2$ is shown in Figs. 8, 9, 10, and 11. Let us assume that (A1), (A2), (A3), and (A4) are true. Then, these are true for $m^{\prime}=m+1$ as it corresponds to adding one more diagram in Fig. 14. Therefore, Eqs. (A1), (A2), (A3), and (A4) are true.

The vacuum structure of $\langle B\rangle$ is derived from Figs. 15 and 17 as follows: 
(i) $N=4 m-3,(m \geq 2)$ :

$$
\{\underbrace{\overrightarrow{1}, \overrightarrow{3}, \ldots, \overrightarrow{4 m-5}, \overrightarrow{4 m-3}}_{2 m}\} \leftarrow\langle B\rangle \leftarrow\{\underbrace{\overrightarrow{2}, \overrightarrow{4}, \ldots, \overrightarrow{4 m-6}, \overrightarrow{4 m-4}}_{2 m}\} .
$$

(ii) $N=4 m-2,(m \geq 2)$ :

$$
\{\underbrace{\overrightarrow{1}, \overrightarrow{3}, \ldots, \overrightarrow{4 m-5}, \overrightarrow{4 m-3}}_{2 m-1}\} \leftarrow\langle B\rangle \leftarrow\{\underbrace{\overrightarrow{2}, \overrightarrow{4}, \ldots, \overrightarrow{4 m-4}, \overrightarrow{4 m-2}}_{2 m-2}\} .
$$

(iii) $N=4 m-1,(m \geq 2)$ :

$$
\{\underbrace{\overrightarrow{1}, \overrightarrow{3}, \ldots, \overrightarrow{4 m-3}, \overrightarrow{4 m-1}}_{2 m}\} \leftarrow\langle B\rangle \leftarrow\{\underbrace{\overrightarrow{2}, \overrightarrow{4}, \ldots, \overrightarrow{4 m-4}, \overrightarrow{4 m-2}}_{2 m-1}\} .
$$

(iv) $N=4 m,(m \geq 2)$ :

$$
\{\underbrace{\overrightarrow{1}, \overrightarrow{3}, \ldots, \overrightarrow{4 m-3}, \overrightarrow{4 m-1}}_{2 m}\} \leftarrow\langle B\rangle \leftarrow\{\underbrace{\overrightarrow{2}, \overrightarrow{4}, \ldots, \overrightarrow{4 m-2}, \overrightarrow{4 m}}_{2 m}\} .
$$

Each case with $m=1$ is shown in Fig. 1. Each case with $m=2$ is shown in Figs. 8, 9, 10, and 11. Let us assume that (A5), (A6), (A7), and (A8) are true. Then, these are true for $m^{\prime}=m+1$ as it corresponds to adding one more diagram in Fig. 15. Therefore, Eqs. (A5), (A6), (A7), and (A8) are true.

For any $N$, the vacuum structure is

$$
\vec{N} \leftarrow \cdots \leftarrow\langle A\rangle \leftarrow \cdots \leftarrow\langle B\rangle \leftarrow \cdots \leftarrow \vec{N} .
$$

Therefore, Eqs. (4.11), (4.12), (4.13), and (4.14) are proven.

[1] P. Di Vecchia and S. Ferrara, Nucl. Phys. B130, 93 (1977); E. Witten, Phys. Rev. D 16, 2991 (1977); A. D’Adda, P. Di Vecchia, and M. Luscher, Nucl. Phys. B152, 125 (1979); B. Zumino, Phys. Lett. 87B, 203 (1979).

[2] L. Alvarez-Gaume and D. Z. Freedman, Commun. Math. Phys. 80, 443 (1981).

[3] T. L. Curtright and D. Z. Freedman, Phys. Lett. 90B, 71 (1980); 91B, 487 (1980); L. Alvarez-Gaume and D.Z. Freedman, Phys. Lett. 94B, 171 (1980); M. Rocek and P. K. Townsend, Phys. Lett. 96B, 72 (1980); U. Lindstrom and M. Rocek, Nucl. Phys. B222, 285 (1983); N. J. Hitchin, A. Karlhede, U. Lindstrom, and M. Rocek, Commun. Math. Phys. 108, 535 (1987); A. S. Galperin, E. A. Ivanov, V. I. Ogievetsky, and E. Sokatchev, Ann. Phys. (N.Y.) 185, 22 (1988).

[4] L. Alvarez-Gaume and D. Z. Freedman, Commun. Math. Phys. 91, 87 (1983).

[5] E. R. C. Abraham and P. K. Townsend, Phys. Lett. B 291, 85 (1992); 295, 225 (1992).

[6] J. P. Gauntlett, D. Tong, and P. K. Townsend, Phys. Rev. D 63, 085001 (2001).

[7] J. P. Gauntlett, D. Tong, and P. K. Townsend, Phys. Rev. D 64, 025010 (2001).

[8] Y. Isozumi, M. Nitta, K. Ohashi, and N. Sakai, Phys. Rev. Lett. 93, 161601 (2004).

[9] Y. Isozumi, M. Nitta, K. Ohashi, and N. Sakai, Phys. Rev. D 70, 125014 (2004).

[10] M. Arai and S. Shin, Phys. Rev. D 83, 125003 (2011).
[11] B. H. Lee, C. Park, and S. Shin, Phys. Rev. D 96, 105017 (2017).

[12] L. K. Hua, Am. J. Math. 66, 470 (1944); 66, 531 (1944); E. Calabi and E. Vesentini, Ann. Math. 71, 472 (1960); S. Kobayashi and K. Nomizu, Foundations of Differential Geometry (Wiley Interscience, New Jersey, 1996), Vol. II.

[13] K. Higashijima and M. Nitta, Prog. Theor. Phys. 103, 635 (2000).

[14] K. Higashijima, T. Kimura, and M. Nitta, Nucl. Phys. B623, 133 (2002).

[15] M. Eto, T. Fujimori, S. B. Gudnason, Y. Jiang, K. Konishi, M. Nitta, and K. Ohashi, J. High Energy Phys. 12 (2011) 017.

[16] A. Isaev and V. Rubakov, Теория групп и симметрий: Конечные группы. Группы и алгебры Ли (KRASAND/ URSS, Moscow, 2017), pp. 504.[Theory of Groups and Symmetries: Finite Groups, Lie Groups, and Lie Algebras (World Scientific, Singapore, 2018), pp. 476].

[17] N. Sakai and D. Tong, J. High Energy Phys. 03 (2005) 019.

[18] J. Wess and J. Bagger, Supersymmetry and Supergravity (Princeton University, Princeton, NJ, 1992), p. 259.

[19] J. Scherk and J. H. Schwarz, Phys. Lett. 82B, 60 (1979); Nucl. Phys. B153, 61 (1979).

[20] E. Witten, Nucl. Phys. B202, 253 (1982); K. Hori and C. Vafa, arXiv:hep-th/0002222; C. U. Sanchez, A. L. Cali, and J. L. Moreschi, Geometriae Dedicata 64, 261 (1997); S. B. Gudnason, Y. Jiang, and K. Konishi, J. High Energy Phys. 08 (2010) 012. 